\title{
Dissection of Ramularia Leaf Spot Disease by Integrated Analysis of Barley and Ramularia collo-cygni Transcriptome Responses
}

\author{
Elisabet Sjokvist, ${ }^{1,2}$ Rene Lemcke, ${ }^{3}$ Manoj Kamble, ${ }^{4}$ Frances Turner, ${ }^{5}$ Mark Blaxter, ${ }^{2}$ Neil H. D. Havis, ${ }^{1}$ \\ Michael F. Lyngkjær, ${ }^{3,+}$ and Simona Radutoiu ${ }^{4,+}$ \\ ${ }^{1}$ Scotlands Rural College, The University of Edinburgh, West Mains Road, Edinburgh EH9 3JG, Scotland, U.K.; ${ }^{2}$ Institute of \\ Evolutionary Biology, The University of Edinburgh, Edinburgh EH9 3JT, U.K.; ${ }^{3}$ Department of Plant and Environmental \\ Sciences, Copenhagen University, Thorvaldsensvej 40, 1871 Frederiksberg C, Denmark; ${ }^{4}$ Department of Molecular Biology and \\ Genetics, Aarhus University, Gustav Wieds vej 10, Aarhus, Denmark; and ${ }^{5}$ Edinburgh Genomics, School of Biological Sciences, \\ The University of Edinburgh; Scotland, U.K.
}

Accepted 10 July 2018.

Ramularia leaf spot disease (RLS), caused by the ascomycete fungus Ramularia collo-cygni, has emerged as a major economic disease of barley. No substantial resistance has been identified, so far, among barley genotypes and, based on the epidemiology of the disease, a quantitative genetic determinacy of RLS has been suggested. The relative contributions of barley and $R$. collo-cygni genetics to disease infection and epidemiology are practically unknown. Here, we present an integrated genome-wide analysis of host and pathogen transcriptome landscapes identified in a sensitive barley cultivar following infection by an aggressive $R$. collo-cygni isolate. We compared transcriptional responses in the infected and noninfected leaf samples in order to identify which molecular events are associated with RLS symptom development. We found a large proportion of $R$. collo-cygni genes to be expressed in planta and that many were also closely associated with the infection stage. The transition from surface to apoplastic colonization was associated with downregulation of cell wall-degrading genes and upregulation of nutrient uptake and resistance to oxidative stresses. Interestingly, the production of secondary metabolites was dynamically regulated within the fungus, indicating that $R$. collo-cygni produces a diverse panel of toxic compounds according to the infection stage. A defense response against $R$. collo-cygni was identified in barley at the early, asymptomatic infection and colonization stages. We found activation of ethylene signaling, jasmonic acid signaling, and phenylpropanoid and flavonoid pathways to be highly induced, indicative of a classical response to necrotrophic pathogens. Disease development was found to be associated with gene

\section{E. Sjokvist and R. Lemcke contributed equally.}

Raw sequence data have been deposited in the ENA read archive under BioProject PRJEB14791.

${ }^{\dagger}$ Corresponding authors: Simona Radutoiu; E-mail: radutoiu@mbg.au.dk and Michael Lyngkjær; E-mail: mlyn@plen.ku.dk

Funding: This study was financed by the Innovation Fund Denmark, grant number 1308-00013B.

*The $\boldsymbol{e}$-Xtra logo stands for "electronic extra" and indicates that 27 supplementary figures and six supplementary tables are published online.

(c) 2019 The American Phytopathological Society expression patterns similar to those found at the onset of leaf senescence, when nutrients, possibly, are used by the infecting fungus. These analyses, combining both barley and $R$. collocygni transcript profiles, demonstrate the activation of complex transcriptional programs in both organisms.

Coevolution of plants and fungi has led to sophisticated mechanisms for mutual recognition, manipulation, and control by the interacting partners (Zeilinger et al. 2016; Zipfel and Oldroyd 2017). Often, fungi inhabiting plants benefit the host or do not cause visible disease symptoms. This endophytic colonization may encompass most of their life cycle (Fesel and Zuccaro 2016). However, plant-fungal interactions are dynamic and under continuous evolution, therefore, the balance between a beneficial and pathogenic outcome is, in many cases, very subtle and dependent on macro- and microenvironmental conditions (Schulz and Boyle 2005). Communications between the plant host and infecting fungus at molecular and cellular levels plays an important role during establishment of their association. Plants recognize fungi present on their surface or in the apoplastic space based on their unique pathogen-associated molecular patterns (PAMPs) through receptors (pattern recognition receptors [PRRs]) localized on the plasma membrane (Choi and Klessig 2016; Zipfel and Oldroyd 2017). If the fungus is pathogenic, either fungal molecules in the apoplastic space or their damaging activity on plant structures are detected via PRRs and a pathogen-triggered immunity (PTI) is induced (Jones and Dangl 2006). Fungi have evolved the capacity to both overcome and interfere with PTI by secreting effectors (Cook et al. 2015). In turn, plants have evolved specific receptors for recognition of these effectors or their activity on host components. Activation of these receptors initiates the effector-triggered immunity (ETI) response (Jones and Dangl 2006). Major changes in transcriptome, proteome, metabolome, and structural components are induced in the plant and the interacting fungi, as part of PTI and ETI responses (Bigeard et al. 2015; Hurley et al. 2014; Li et al. 2016).

Ramularia collo-cygni is an ascomycete fungus commonly associated with wild grasses and cereal crops, including wheat, oat, rye, maize, and barley (Huss 2004). Sensitive polymerase chain reaction (PCR)-based methods (Havis et al. 2006) have confirmed the worldwide presence of $R$. collo-cygni in asymptomatic tissues, including seeds, implying an endophytic 
and highly dispersive life style (Havis et al. 2015a). R. collocygni is the causal agent of Ramularia leaf spot (RLS) disease in barley (Sutton and Waller 1988). RLS has emerged recently as a serious disease of barley in Europe as well as most other temperate regions (Walters et al. 2008). The disease leads to major reductions in green leaf area and up to $20 \%$ yield losses, depending on the environmental conditions and barley genotype, highlighting the necessity for improved RLS resistance in barley (Havis et al. 2015b; Walters et al. 2008). Testing for RLS resistance in a wide range of winter and spring barley cultivars (Havis et al. 2015b) failed to identify barley genotypes with substantial resistance. Furthermore, no clear division between resistant and susceptible cultivars was observed, suggesting that RLS resistance is generally a quantitative trait. The nature and molecular mechanisms behind resistance and susceptibility traits are mainly unknown. The only genetic factor influencing RLS severity described so far is the barley Mlo gene. This gene is required for susceptibility to powdery mildew (Blumeria graminis) and mutated nonfunctional (mlo) alleles conferring highly effective resistance against powdery mildew disease have been widely used in elite European spring barley (Acevedo-Garcia et al. 2014). However, carrying an mlo mutation has been associated with enhanced susceptibility against some facultative pathogens, including $R$. collo-cygni. The reason for this is currently unknown (McGrann et al. 2014).

Major advances have been made over the last years in precise $R$. collo-cygni detection, $R$. collo-cygni infection biology and epidemiology (Havis et al. 2015a; Kaczmarek et al. 2017; Taylor et al. 2010; Thirugnanasambandam et al. 2011), and $R$. collo-cygni natural diversity (Hjortshøj et al. 2013). Detailed infection studies using scanning electron microscopy of field samples (Stabentheiner et al. 2009) or confocal microscopy of artificially infected barley leaves with fluorescently labeled $R$. collo-cygni isolates (Thirugnanasambandam et al. 2011) revealed that foliar infection starts with conidial adhesion and germination on green leaves. This is followed rapidly by the development of a hyphal network formation and symptomless invasion of the mesophyll apoplast via stomatal pores. $R$. collocygni can complete its infection cycle and produce spores in various hosts, including barley, without producing any disease symptoms. However, in barley, R. collo-cygni tends to switch from asymptomatic endophytic colonization to pathogenic growth at the time of ear emergence, and flowering. RLS symptom development is followed by prolific sporulation on the leaf abaxial surface. Initially, visual RLS symptoms resemble small pepper spots that, over time, develop into diseasespecific rectangular necrotic lesions surrounded by a chlorotic halo, bounded by leaf veins and visible on both the adaxial and abaxial leaf surfaces. Eventually, depending on the environmental conditions, symptoms may merge to cover the entire leaf, reducing photosynthetic area and assimilate production. RLS disease symptoms are associated with massive $R$. collocygni sporulation from the abaxial side of leaves late in the cropping season (Walters et al. 2008).

Genome sequencing of $R$. collo-cygni (isolate DK05 Rcc001) confirmed its phylogenetic position within the class Dothidiomycetes and family Mycosphaerellaceae (McGrann et al. 2016). Closely related fungal species such as Zymoseptoria tritici, Pseudocercospora fijiensis, and Dothistroma septosporum are major pathogens of wheat, banana, and pine tree, respectively, and, like many known Dothidiomycetes pathogens, use toxic secondary metabolites (SM) to control their hosts (Muria-Gonzalez et al. 2015). The R. collo-cygni genome has multiple genes predicted to be involved in toxin metabolism (McGrann et al. 2016), and R. collo-cygni is known to produce photoactivated anthraquinone toxins called rubellins (Heiser et al. 2003). Rubellin D has been shown to induce fatty acid peroxidation leading to leaf chlorosis and necrosis (Heiser et al. 2004), but its specific role in $R$. collo-cygni pathogenesis in barley remains unclear.

Here, to advance understanding of RLS disease, which has a growing economic importance, we provide a comprehensive analysis of the molecular events associated with transcriptome reprogramming in barley and $R$. collo-cygni during their pathogenic association.

\section{RESULTS}

\section{Disease progression of RLS in barley in an experimental infection.}

In this study, we investigated time-resolved transcriptional changes induced in barley cv. Fairytale and $R$. collo-cygni isolate DK05 during RLS symptom development. Leaves of control, uninfected plants and plants infected with DK05 were inspected visually daily, and samples were collected at 3, 7, and 12 days postinfection (dpi) for histological and molecular analyses. Quantitative measurements of relative $R$. collo-cygni DNA content in the infected samples revealed an increase in fungal biomass over time, despite the lack of visible RLS symptoms until $11 \mathrm{dpi}$, when the first small necrotic spots were observed (Fig. 1A and B). Histological examination of inoculated leaf samples confirmed that $R$. collo-cygni infection occurred through penetration of stomata openings (Sutton and Waller 1988). Only sporadic stomatal penetrations were observed at $3 \mathrm{dpi}$, whereas these were more frequent at 7 and 12 dpi (Fig. 1C). No visible macroscopic host responses were observed at 3 and 7 dpi. However, at 12 dpi, local browning of mesophyll cells frequently appeared below stomata penetrated by fungal hyphae, corresponding to the necrotic symptoms visible at this time point (Fig. 1). RLS symptoms developed rapidly thereafter, with increasing size of the necrotic spots and leaf chlorosis leading to complete leaf death at 17 dpi (Supplementary Fig. S2). No disease symptoms were detected on the noninoculated control leaves, which remained healthy and green throughout the experiment.

\section{Dataset integrity.}

A total of 1.565 billion paired RNA-seq reads were obtained (5.0 to 9.8 million per sample), and these were mapped to the combined genomes of barley and $R$. collo-cygni (Supplementary Table S1). Over $80 \%$ of the reads mapped with high mapping quality to a single location across all samples, while a maximum of $5 \%$ remained unmapped and a maximum of $14.7 \%$ mapped to multiple locations (Supplementary Fig. S3). As expected, in the infected samples, most reads mapped to the barley genome (Supplementary Table S2). In general, the number of $R$. collo-cygni reads, as a fraction of the total, increased over the course of the infection, reflecting the increase in fungal biomass (Fig. 1B). Reads mapping to R. collo-cygni reached a maximum of $1.3 \%$ in one of the 12-dpi samples. The level of sequence saturation obtained was determined by identification of known and novel exon junctions (Supplementary Fig. S4). For barley, nearly $40 \%$ of the reads mapped to novel junctions, and, overall, the barley transcriptome was sequenced at sufficient depth, as full saturation was observed. For $R$. collo-cygni, for which fewer reads were obtained, we identified 20\% novel splice junctions and, based on the saturation curve, we found these to be sufficiently covered. In contrast to barley, the $R$. collo-cygni reads did not cover sufficiently the known $R$. collo-cygni splice junctions, most likely due to the lower prevalence of $R$. collo-cygni transcripts in our samples when compared with barley. 
Analysis of data integrity based on multidimensional scaling revealed that biological replicates grouped together and that, for both barley and $R$. collo-cygni datasets, the time point of analysis was the main differentiating driver (Supplementary Fig. S5). Interestingly, we found that, with one exception (one biological replica of $R$. collo-cygni-infected barley at 7 dpi), the infected and control barley samples clustered together at 3 and $7 \mathrm{dpi}$, whereas a clear separation between them was observed at 12 dpi.

\section{R. collo-cygni infection reprograms the barley transcriptome.}

Analyses of barley transcripts from uninfected, control samples revealed a response to time between the 3-, 7-, and 12day analyses points (Fig. 2A to C). In the $R$. collo-cygni-treated samples, we detected a clear response to fungal infection and colonization based on the transcriptional landscape present in control and infected samples at the corresponding time points (Fig. 2D to F). We identified an increasing number of statistically significant differentially expressed genes (DEGs) in inoculated versus control leaves from 3 to 12 dpi (Fig. $2 \mathrm{G}$ and $\mathrm{H}$ ), indicating reprogramming of the barley transcriptome during the different stages of infection. Overall, of the 39,471 highconfidence barley genes (Mascher et al. 2017), 5,575 were found to be differentially expressed after $R$. collo-cygni inoculation, with a similar number of significant upregulated $(n=2,731)$ and downregulated $(n=2,902)$ genes (Fig. $2 \mathrm{G}$ and $\mathrm{H})$. However, when analyzing the individual time points, we found a prevalence of upregulation at 3 and $7 \mathrm{dpi}$, whereas, at 12 dpi, more genes were downregulated (Fig. 2G and $\mathrm{H}$ ). Interestingly, only 360 genes were significantly up- or downregulated throughout the infection period, with a majority of them $(n=328)$ being upregulated. Twenty-two genes were continually downregulated and eight had variable relative expression profiles during the experimental infection process.

\section{$R$. collo-cygni infection in barley triggers a pathogenic response detected at the molecular level.}

Molecular responses associated with senescence are detected during R. collo-cygni infection. $R$. collo-cygni infection of barley is recognized by the induction of necrotic pepper spots and leaf chlorosis symptoms. In healthy plants chlorosis is associated with senescence. Therefore, we have explored our data for molecular signatures associated with senescence by analyzing the expression profiles of genes previously shown to respond to senescence in barley (Kucharewicz et al. 2017). Among 28 reported marker genes from five functional categories important in senescence (transcription factor [TF], photosynthesis-, stress-, remobilization-, and degradation-related genes) most showed a similar regulation in response to $R$. collocygni inoculation at 12 dpi (Supplementary Table S3). This indicates that $R$. collo-cygni infection induces premature leaf senescence in barley.

$\mathrm{R}$. collo-cygni infection leads to regulation of specific processes and functions in barley leaves. The robust transcriptional response to $R$. collo-cygni infection prompted us to identify whether specific biological processes, cellular components, and molecular functions are particularly enriched in
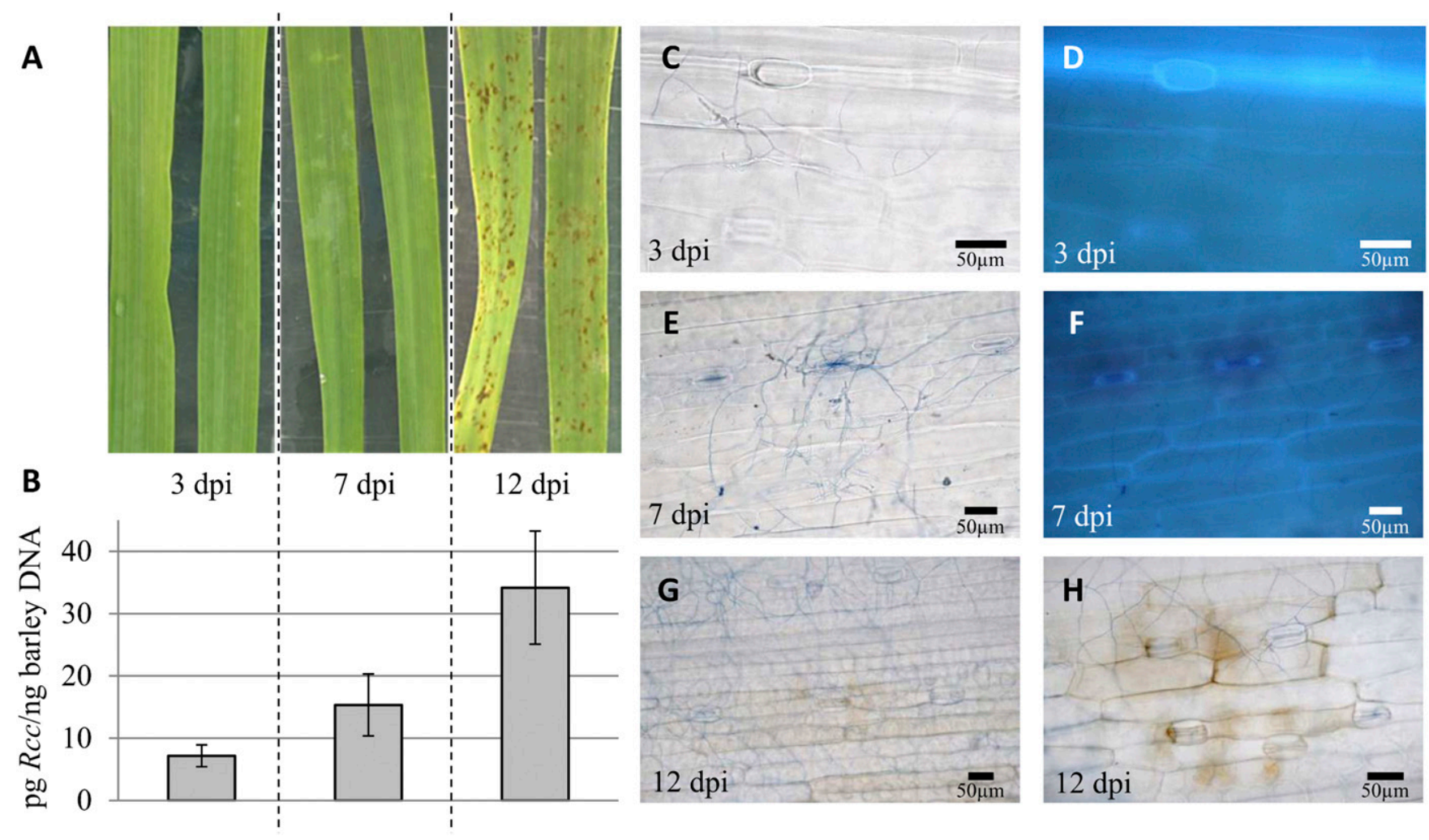

Fig. 1. The progress of Ramularia leaf spot (RLS) disease on leaves of barley (Hordeum vulgare L.) cv. Fairytale inoculated with Ramularia collo-cygni DK05. A, Development of RLS disease symptoms at 3, 7, and 12 days postinfection (dpi). B, Relative content of $R$. collo-cygni DNA in infected leave samples at 3, 7, and 12 dpi. C, Light micrograph of $R$. collo-cygni hyphae on the barley leaf surface at 3 dpi, showing limited fungal growth and no visible host cell responses. D, Incident fluorescence micrograph of the same cells seen in C. E, Light micrograph of $R$. collo-cygni hyphae on the barley leaf surface at $7 \mathrm{dpi}$, showing increased fungal growth but no visible host cell responses. F, Incident fluorescence micrograph of the same cells seen in C, showing no visible host cell responses. G, Light micrograph of $R$. collo-cygni hyphae on the barley leaf surface at $12 \mathrm{dpi}$, showing extensive fungal growth and local browning of host cells corresponding to the necrotic leaf spots. H, Closeup of same leaf segment as shown in G, showing browning of epidermal cells and underlying mesophyll cell next to stomata penetrated by fungal hyphae. 
the up- and downregulated DEGs at specific time points, based on their gene ontology (GO) annotation. Analysis of enriched biological processes revealed three major groups (Fig. 3, designated A, B, and C). The first group (A) contained primarily metabolic processes that are enriched in both down- and upregulated genes and included modification of proteins and transport process functions. These metabolic processes are enriched among genes upregulated at all time points and among those downregulated at $12 \mathrm{dpi}$. The second group (B) contained primarily DNA/RNA metabolic processes and photosynthesis was enriched, primarily among the 12-dpi downregulated DEGs, while the third group (C), containing transport and metabolic processes, was specifically enriched in the 12-dpi upregulated DEGs. A similar pattern was observed for the GOs with molecular function terms. Major molecular processes linked to plant cell activity (A) were enriched in the 12-dpi downregulated genes, likely corresponding to the physiological responses and the disease symptoms development (Fig. 1)
Transmembrane transporter activity, kinase activity, and catalytic activities such as lyase or ATPases were enriched in the upregulated DEGs $(\mathrm{C})$. These findings suggest reprogramming of vital molecular and biological functions throughout the infection period indicative of a rapid and continuing response to $R$. collo-cygni infection, despite the visually symptomless infection and colonization at 3 and 7 dpi (Fig. 1).

Based on the findings from GO enrichment analyses and previous identification of classes of plant genes regulated during pathogen infection (Palma-Guerrero et al. 2016; Powell et al. 2017), we focused our detailed investigation on DEGs encoding proteins with molecular functions covering cell-wall processes, receptor-like kinases (RLKs), transmembrane transport, TFs, oxi-reductases, hormones, defense genes, and phenolic compound synthesis. A subset of these (with log fold change $[\mathrm{FC}]>2$ or $<-2$ ) are illustrated in Figure 4.

Cell wall-related processes are primarily regulated at later stages of infection. Sixty-two barley genes predicted to be

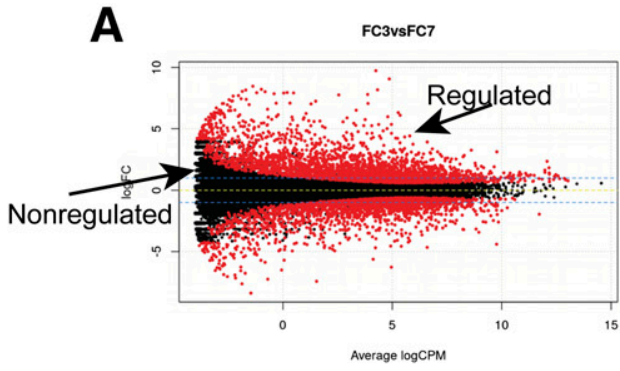

D

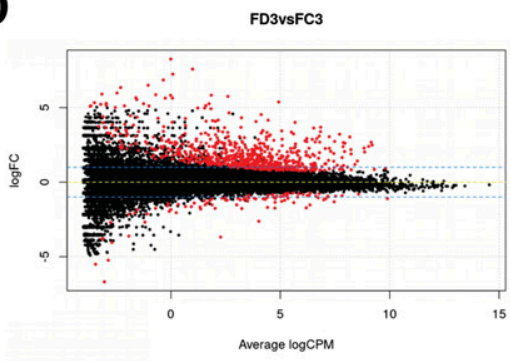

G

Upregulated DE genes

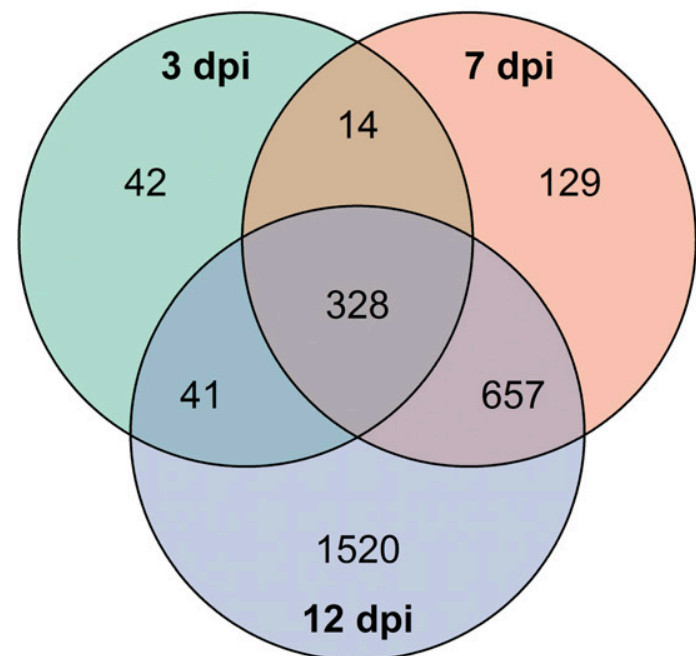

B

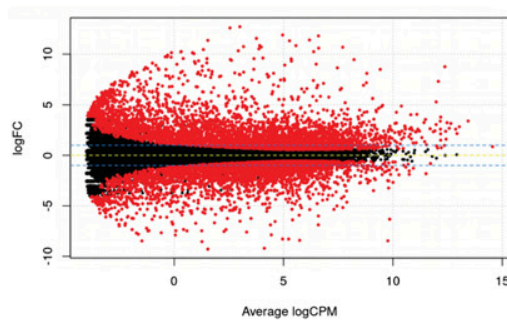

E

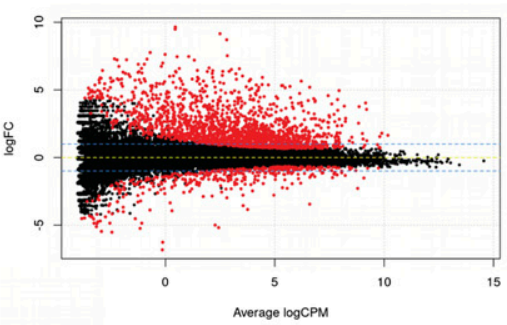

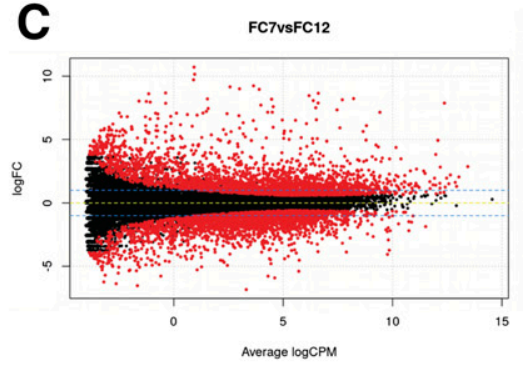

$\mathbf{F}$

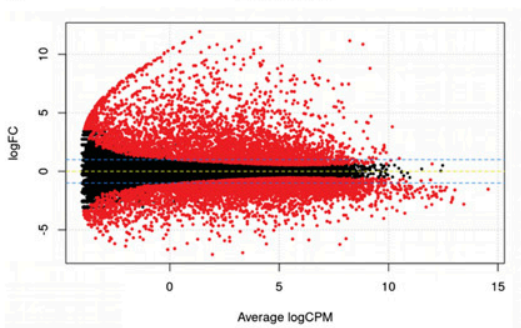

H

\section{Downregulated DE genes}

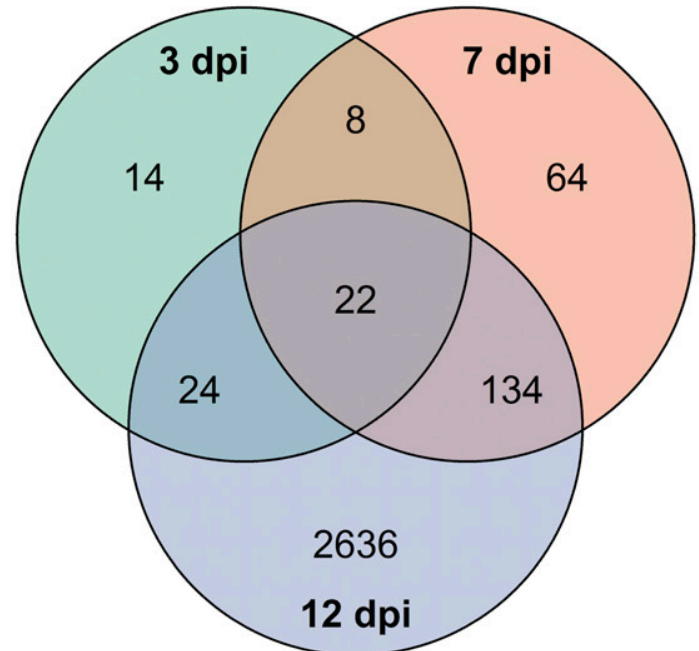

Fig. 2. Differentially expressed (DE) barley genes during Ramularia leaf spot (RLS) disease progression. A to F, The distribution of DEGs and non-regulated genes when comparing noninfected barley leaves at the analyzed time points (A to C) and when comparing infected (FD) versus noninfected (FC) barley leaves at 3 (D), 7 (E), and 12 (F) days postinoculation. Each individual gene is plotted according to the fold change and the average expression (log counts per million). $\mathbf{G}$ and $\mathbf{H}$, Venn diagram of differentially expressed barley genes at the analyzed time points according to their up- or downregulation pattern. 
involved in cell-wall processes, such as modification, degradation, pectin esterases, and synthesis of cellulose, hemicellulose, pectins, and precursors of this, were found among the DEGs. Interestingly, only two of these are regulated at 3 dpi, both being downregulated (HORVU4Hr1G069100 and
HORVU1Hr1G054240 [Fig. 4]). An increasing number of DEGs predicted to encode enzymes involved in cell-wall processes were found at 7 and 12 dpi (Fig. 4), indicating increasing importance of activity at the cell-wall interface during mesophyll colonization by $R$. collo-cygni and necrotic

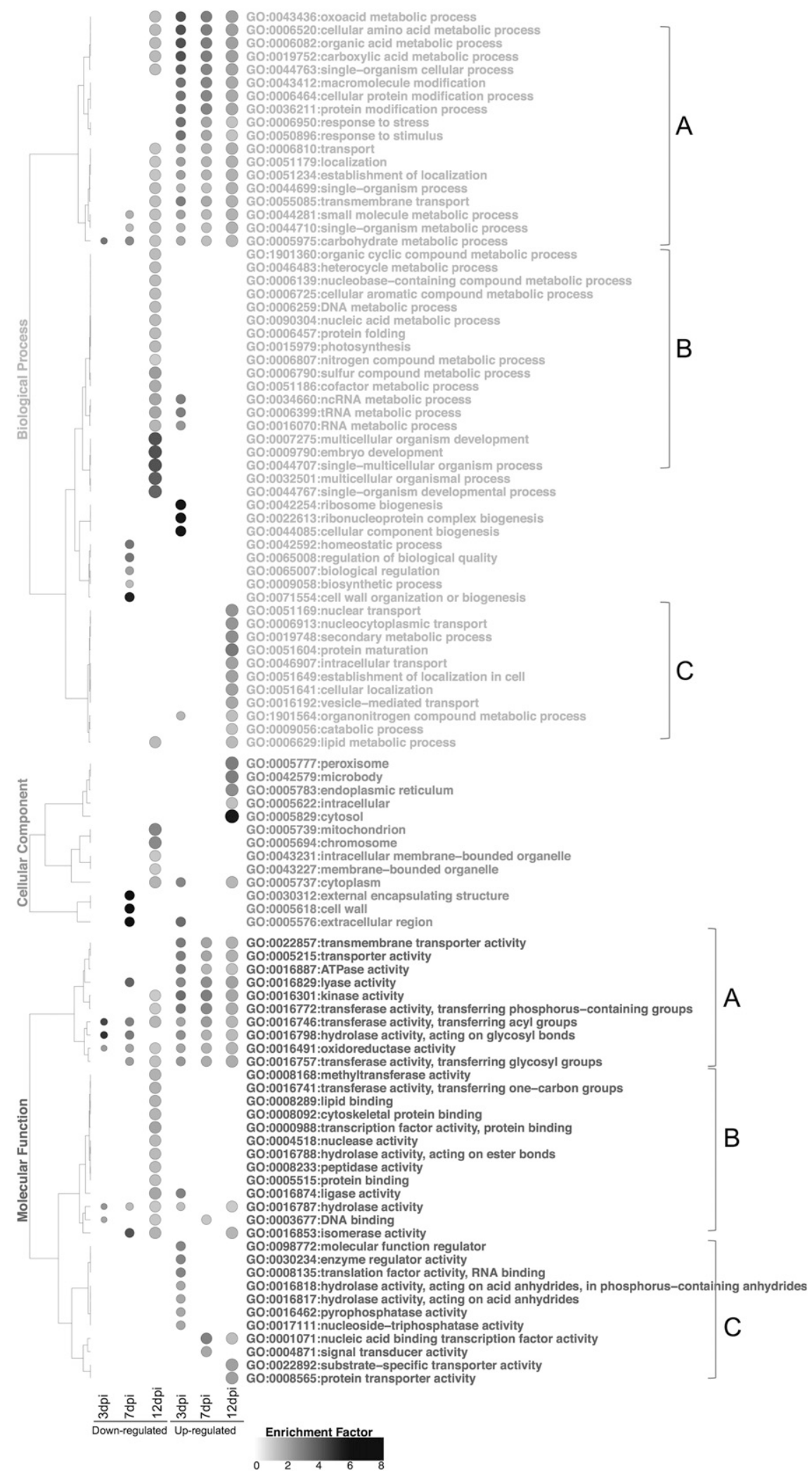

Fig. 3. Enriched functions based on gene ontology (GO) analyses of differentially expressed barley genes at the analyzed time points (3, 7, and 12 days postinoculation), grouped based on their down- or upregulation pattern. The shades of the circles is adjusted according to the enrichment factor and the size is adjusted according to the number of genes present in each GO category. Note the enrichment of specific biological and molecular functions in the marked A, B, C groups. 
symptom development. Several genes predicted to be involved in cell-wall modification were down-regulated at 7 and $12 \mathrm{dpi}$, as were pectin esterases, while genes involved in cellulose and hemicellulose synthesis were upregulated at 12 dpi (Supplementary Fig. S6).

A large number of genes encoding $R L K s$ are regulated during $\mathrm{R}$. collo-cygni infection. The RLK category was one of the largest categories of regulated DEGs in response to $R$. collocygni $(n=338$ genes). However, most RLK genes had a low FC of expression across time points (note the 87 genes grouped according to their predicted extracellular domain [Fig. 4]). CRKs, leucine-rich repeat (LRR) kinases, and a LysM receptor similar to rice CERK1 were strongly induced throughout infection (Fig. 4). The overall pattern of expression observed for RLKs was upregulation at 3 and 7 dpi (Supplementary Fig. S7), while downregulation was primarily observed at $12 \mathrm{dpi}$, at the later stage of the infection (Fig. 4).

Genes encoding transmembrane transporters are highly induced throughout $\mathrm{R}$. collo-cygni infection. Many genes $(n=$ $373)$ predicted to encode transmembrane transporters were found differentially expressed during $R$. collo-cygni infection. These genes were grouped according to their potential substrate into transporters of amino acids and peptides, inorganic cation and anion, SM, sugar, and others (Fig. 4; Supplementary Fig. S8). Predicted metabolite transporters, such as ABC $(n=8)$, MATE $(n=4)$, and peptide transporters such as NRT1/PTR family transporters $(n=5)$, were found to be highly upregulated throughout infection and showed a strong upregulation at 3 dpi (Fig. 4). Interestingly, most of the genes encoding transporters (95\%) upregulated at 3 dpi continued to be strongly upregulated at 7 and $12 \mathrm{dpi}$ and $90 \%$ of those induced at $7 \mathrm{dpi}$ continued to be strongly induced at $12 \mathrm{dpi}$, indicating a continuous need for these transporters throughout $R$. collo-cygni infection.

TFs coordinating the observed molecular responses are regulated during $\mathrm{R}$. collo-cygni infection. We identified 220 genes encoding typical defense-associated TFs to be specifically regulated during the infection experiment (Fig. 4). They included basic helix-loop-helix, MYB, NAC, and WRKY classes (Supplementary Fig. S9). TFs associated with stress responses, such as NAC and WRKY, were found mainly upregulated at 7 and 12 dpi during fungal colonization of the apoplast. TFs were the only group of analyzed genes that, at 12 dpi, contained a larger number of significantly downregulated DEGs $(n=121)$ than upregulated DEGs $(n=81)$, perhaps reflecting host downregulation of major physiological processes as observed at this stage of $R$. collo-cygni infection.

Genes coding for oxi-reductases are highly induced at the early stage of R. collo-cygni infection. Many DEGs $(n=101)$ had predicted oxi-reductase function, including glutathione $S$ transferases (GST), peroxidases, ascorbate and glutathione, and thioredox-reductases. Most of them were upregulated (Fig. 4).
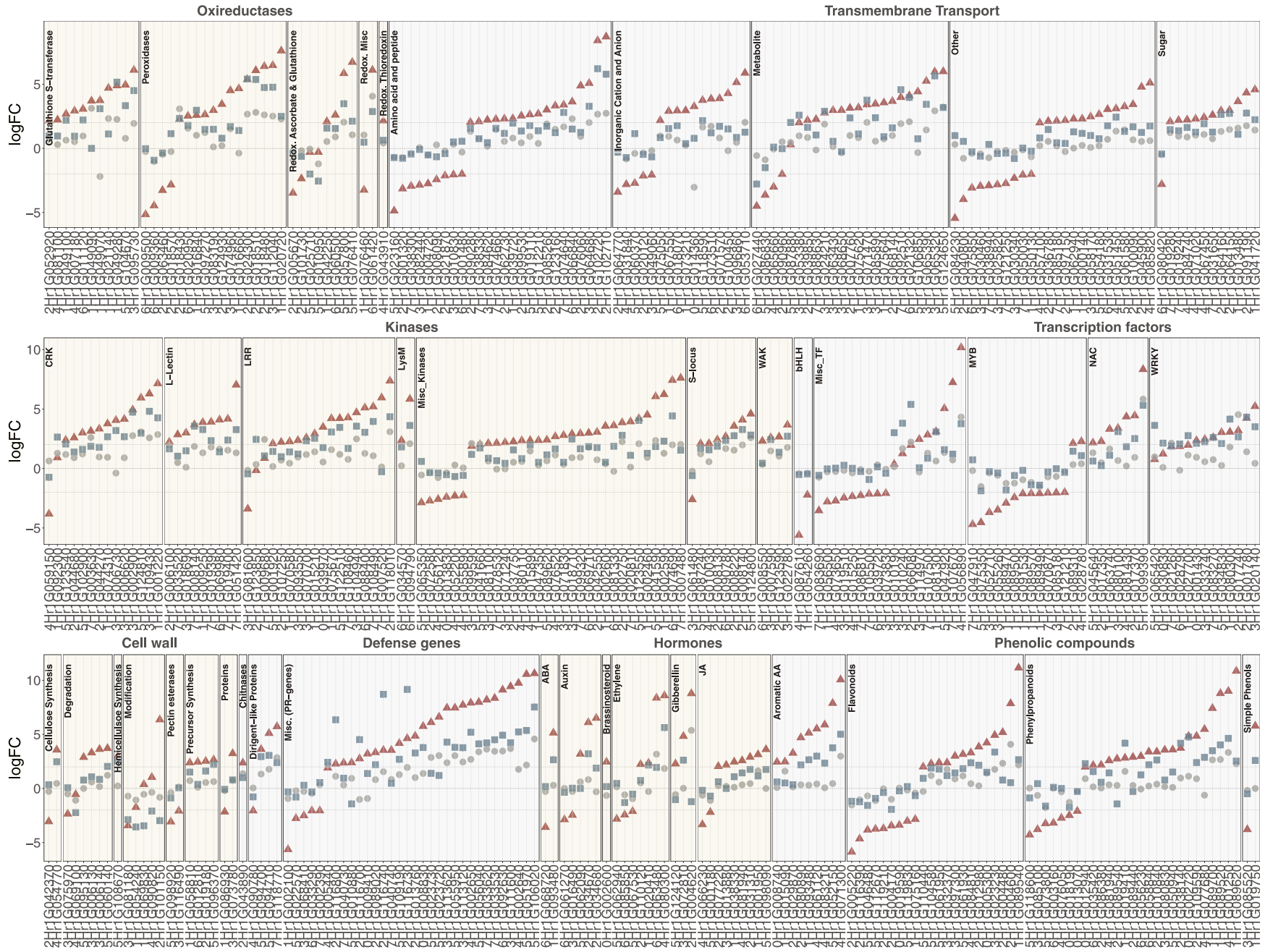

Fig. 4. Selected barley genes with differential expression during RLS development. The log fold change (FC) between infected and uninfected leaf samples at 3 (squares), 7 (circles), and 12 days postinoculation (triangles) for the selected genes grouped according to their predicted functional annotation is shown. The log $2 /-2$ values are marked by horizontal guiding lines. 
Genes encoding GST and peroxidases were found to be highly induced early in infection (from 3 dpi [Supplementary Fig. S10]), indicating activation of host responses during stomata penetration by the fungal hyphae. This would create an environment rich in reactive oxygen species (ROS) necessary for reinforcement of the cell wall but also for reducing fungal infection. Interestingly, the DEGs in the group of thioredoxreductases were found to be primarily downregulated at the late stage of infection.

Regulation of genes involved in phytohormone signaling reflects a necrotrophic behavior during the pathogenic stage of barley-R. collo-cygni association. Several genes $(n=99)$ predicted to be involved in phytohormone metabolism and signaling were found to be regulated during $R$. collo-cygni infection (Figs. 4 and 5A). Genes involved in auxin transport and response and ethylene (ET) response factors were highly induced from $3 \mathrm{dpi}$, indicating early ET and auxin signaling in response to stomata penetration. One DEG involved in gibberellic acid (GA) biosynthesis showed the highest level of upregulation at $3 \mathrm{dpi}$, and a large number of genes $(n=$ 25) involved in jasmonic acid (JA) biosynthesis and signaling were induced from 3 dpi but primarily at 7 and 12 dpi (Supplementary Fig. S11). Meanwhile, only one UDPglycosyltransferase (HORVU5Hr1G079670) potentially related to salicylic acid (SA) metabolism was found to be induced. The Arabidopsis thaliana homolog of this locus, UGT74F1, was previously suggested to be involved in SA glycosylation and, potentially, inactivation of SA signaling (Dean and Delaney 2008). Together, the observed activation of GA, ET, and JA signaling during $R$. collo-cygni infection represent strong indications for a switch to the necrotrophic lifestyle, as previously observed for other necrotrophic fungi (De Vleesschauwer et al. 2014). This is further illustrated by the upregulation of cytokinin (CK) oxidases and of abscisic acid response genes at 7 and $12 \mathrm{dpi}$, indicating a stress response and likely limitation of CK-dependent plant growth.

Defense genes were primarily activated at later stages of R. collo-cygni infection. Pathogenesis-related (PR) genes have been reported as important players in defense responses against invading microbes (Linthorst and Van Loon 1991). We found, in our dataset, many differentially regulated PR genes $(n=85)$ belonging to different categories, including chitinases, dirigentlike proteins, LRR-domain proteins, NB-ARC domain proteins, serpin proteins, and others (Fig. 4). The number of regulated PR genes and their level of expression increased over time. We identified 25 PR genes that were significantly up-regulated at 3 dpi, while none were found to be downregulated. The number of genes upregulated increased to 40 and 52 at 7 and $12 \mathrm{dpi}$, respectively (Supplementary Fig. S12), suggesting a rather limited defense response to $R$. collo-cygni colonization at the early stage of infection. Five $P R$-1-like genes were found to be upregulated at all three time points, with one of them being one of the most upregulated genes in our dataset (HORVU5Hr1G106020) (Supplementary Table S4). Interestingly, four genes predicted to encode wound-responsive proteins (HORVU2Hr1G116730, HORVU2Hr1G116740, HORVU2Hr1G116800, HORVU2Hr1G116700) were found to be upregulated at $7 \mathrm{dpi}$, suggesting a specific response to $R$. collo-cygni colonization at this particular stage of the infection.

Nonfunctional mutations of the barley MLO gene has been proposed to modulate $R$. collo-cygni tolerance (Makepeace et al. 2007; McGrann et al. 2014). We found the MLO gene (HORVU4Hr1G082710) and four MLO-like genes (HORVU0Hr1G008830, HORVU2Hr1G013760, HORVU7Hr1G002390, HORVU6Hr1G033290) were differentially regulated in our dataset. The MLO gene and its homolog had a low upregulation level (one- to threefold) at $7 \mathrm{dpi}$, while one MLO- like gene (HORVU0Hr1G008830) was significantly upregulated from 3 dpi.

$\mathrm{R}$. collo-cygni infection triggers a dynamic regulation of genes involved in phenolic compounds and SM synthesis. Genes involved in SM pathways play important roles in plant defense, and we found many loci with annotations related to SM production $(n=113)$ to be actively regulated in our experiment (Fig. 4; Supplementary Fig. S13). Genes involved in the biosynthesis of aromatic amino acids, simple phenols, and phenylpropanoids (Fig. 5B) were predominantly upregulated while genes predicted to contribute to flavonoid production were both up- and downregulated (Fig. 5C). Genes predicted to encode chalcone synthases were both strongly up- and downregulated, indicating a dynamic flavonoid biosynthesis activity.

\section{$R$. collo-cygni reprograms its transcriptome according to the infection stage.}

$R$. collo-cygni was also found to modulate its transcriptome dynamically during the infection experiment. The published gene models for $R$. collo-cygni DK05 were derived from predicted open reading frames and transcriptome data from in vitro growth (McGrann et al. 2016). Our extensive RNA-seq dataset from in planta growth provided us the opportunity to perform an improved, unsupervised genome annotation. This identified 11,131 gene models. Of these, 4,489 genes were found to have coding sequence identical to a previous model and 4,033 genes to have an overlap with a previous model. Nearly one quarter of the overall identified gene models (2,609 genes) were novel. These new gene predictions were functionally annotated for downstream analyses (Supplementary Table S5).

We took advantage of the existing in vitro transcriptome dataset to perform a comparative analysis with transcripts detected during infection in planta. Approximately 70\% (7668 genes) of predicted $R$. collo-cygni genes were found to be expressed in planta (transcripts per million [TPM] values $>5$ in all biological replicates at a particular infection time point). Nearly half $(5,365$ genes $)$ were expressed at one or more time points in planta $(3,7$, or $12 \mathrm{dpi})$ but were not reported to be expressed during in vitro growth (McGrann et al. 2016). Only 125 genes expressed during in vitro growth were not expressed in planta (Fig. 6D). An increase in the number of $R$. collocygni-expressed genes was found from 3 dpi (4,602 genes) to 7 dpi (5,921 genes) and to 12 dpi (7,209 genes) (Fig. 6D). Overall analyses of $R$. collo-cygni genes identified 991 (at 3 versus $7 \mathrm{dpi}$ ), 798 ( 7 versus $12 \mathrm{dpi}$ ), and 1,042 (3 versus 12 dpi) genes to be significantly regulated between the indicated time points. This demonstrates a continuous reprogramming of the fungal transcriptome during the infection time course (Fig. 6; Supplementary Fig. S14). Only 45 of these DEGs were significantly up-regulated and 10 were down-regulated throughout the in planta analyses (at 3 versus 7 dpi and 7 versus 12 dpi) (Supplementary Table S6). Among the 44 continuously upregulated transcripts, we identified genes involved in toxin metabolism, sugar and potassium transport, oxi-reduction, and transcriptional activation, as well as genes with no functional annotation. A predicted secreted protein, a cell-wall mannoprotein, and genes with no assigned domains were among the 10 genes continuously downregulated.

Many of the regulated $R$. collo-cygni genes encode proteins with no assigned domains (Supplementary Fig. S15) and, consequently, no assigned GO terms. Nevertheless, we investigated the GO annotation of DEGs across the experiment. We found that oxidation-reduction, transmembrane transport, and metabolic processes dominated both the down- and upregulated fungal biological activities across the three time points 


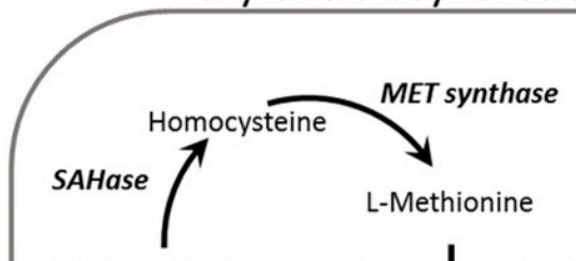

S-Adenosyl-L-homocysteine

MAT
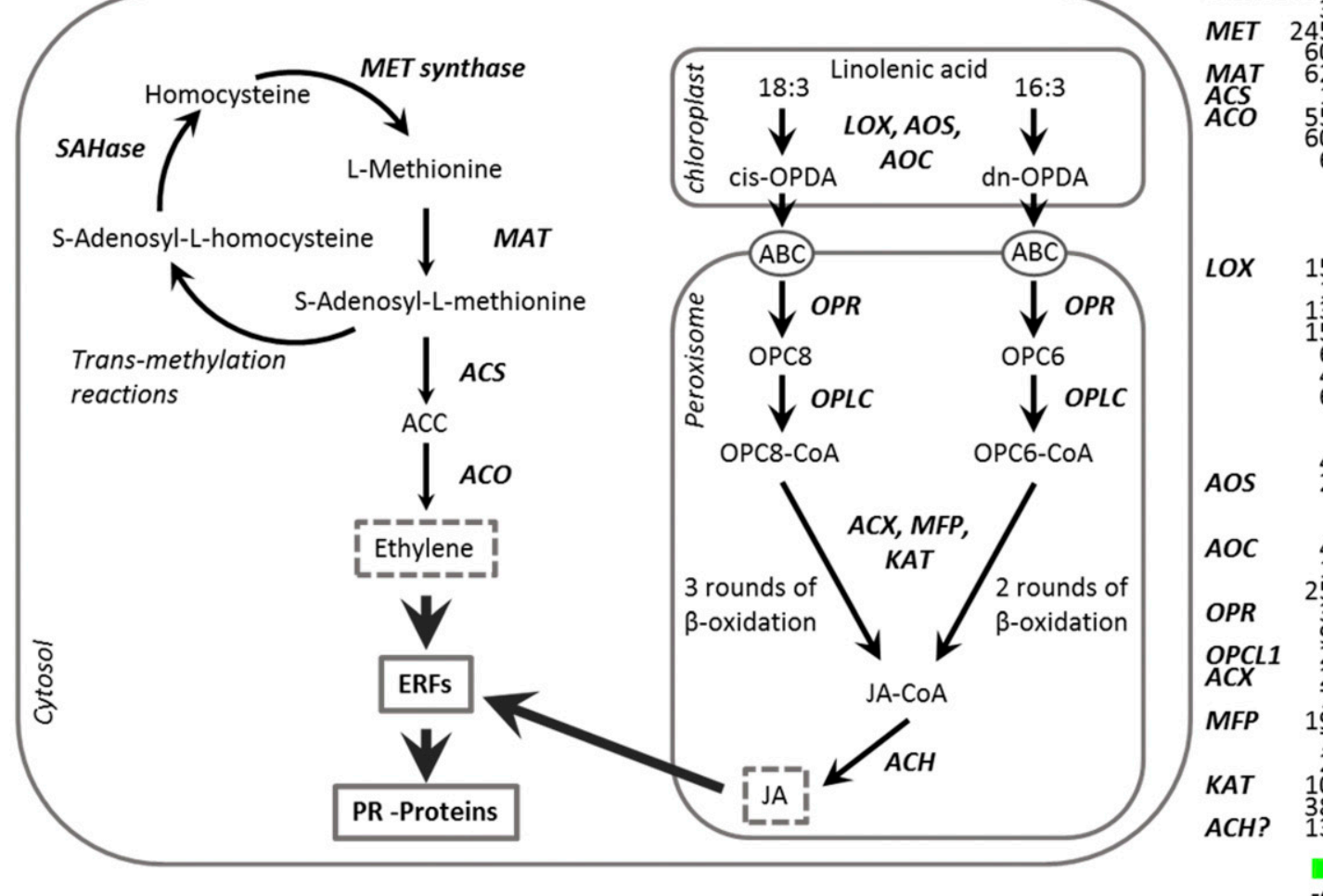

2FD क्त्तु Gene ID
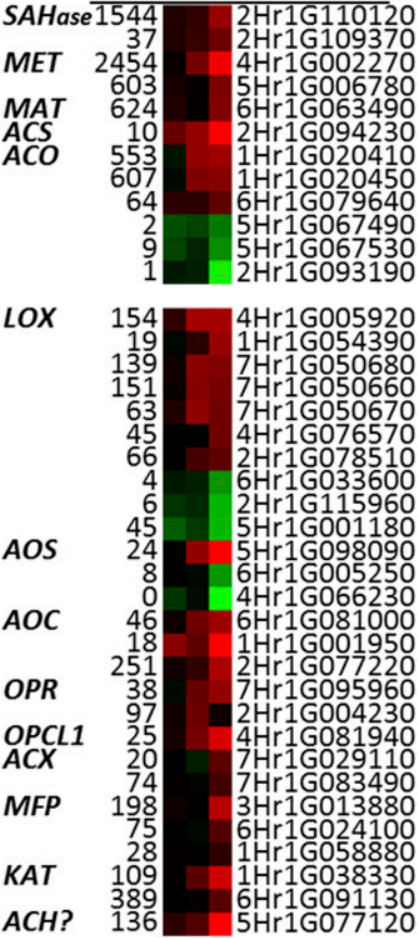

B

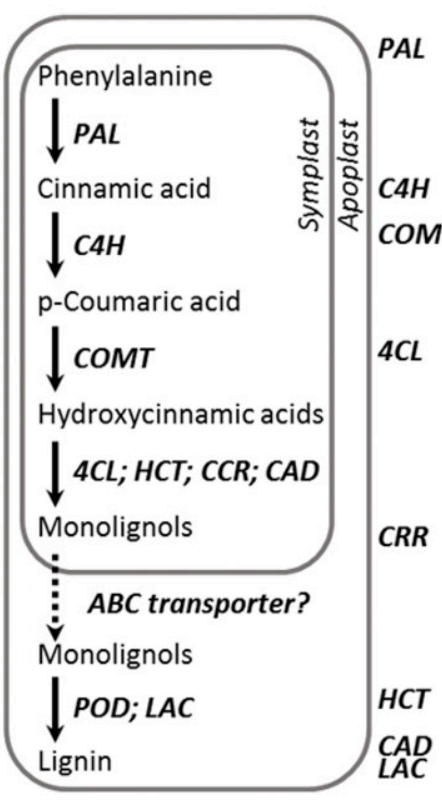

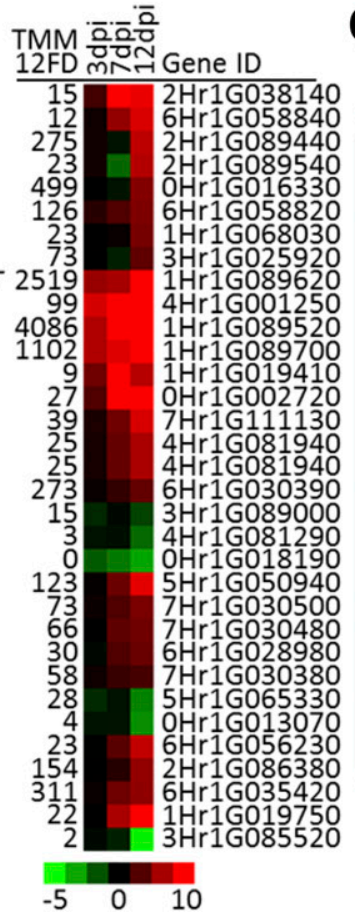

C

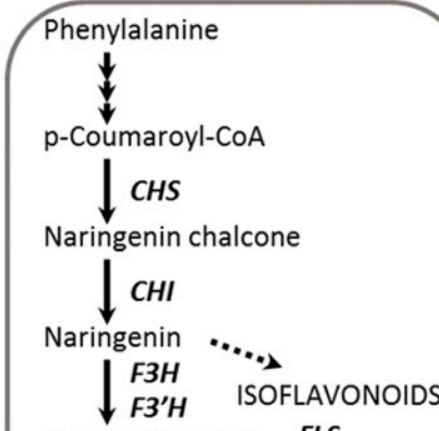

Dihydroflavonols

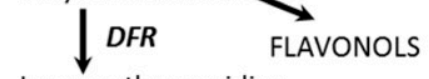

Leucoanthocyanidins
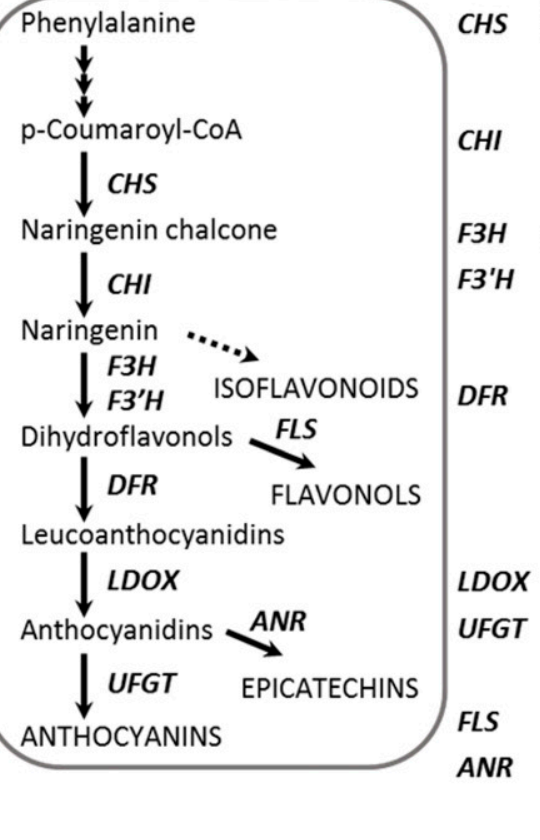

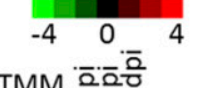

TMM 즈음음

12FD गृत्न Gene ID

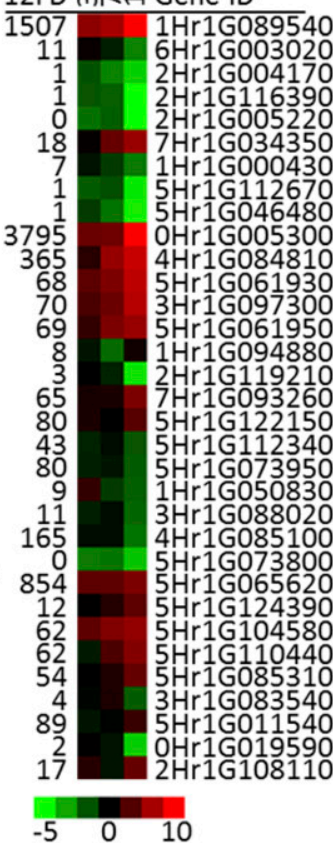

Fig. 5. A, Transcriptional regulation of the ethylene and jasmonate phytohormone, $\mathbf{B}$, phenylpropanoid, and $\mathbf{C}$, flavonoid pathways in barley during Ramularia collo-cygni infection and colonization. Enzymes catalyzing the different steps in the pathways are shown in bold. Expression of the involved genes are listed next to the pathway as trimmed mean method value (abundancy) in $R$. collo-cygni-inoculated leaves at 12 days postinfection (dpi) and as a heatmap showing fold regulation in infected versus control leaves at 3, 7, and 12 dpi. Ethylene and jasmonate pathways: SAHase = adenosylhomocysteinase, MET synthase = cobalamin-independent methionine synthase, MAT = methionine adenosyltransferase, ACS = 1-aminocyclopropane-1-carboxylate synthase, ACO = 1-aminocyclopropane-1-carboxylate oxidase, $\mathrm{ACC}=$ aminocyclopropane-1-carboxylic acid, $\mathrm{LOX}=$ lipoxygenase, $\mathrm{AOS}=$ allene oxide synthase, $\mathrm{AOC}=$ allene oxide cyclase, OPR = oxophytodienoate-reductase, OPCL1 = OPC-8-0 CoA ligasel/4-coumarate-CoAligase, ACX = acyl-coenzyme A oxidase, MFP = 2-transenoylCoA hydratase/1-3-hydroxyacyl-CoA dehydrogenase, KAT = 3-ketoacyl-CoA thiolase, ACH = esterase/lipase/thioesterase family protein. Phenylpropanoids pathway: PAL = phenylalanine ammonia-lyase, $\mathrm{C} 4 \mathrm{H}=$ cinnamate $4=$ hydroxylase $(\mathrm{CYP} 73 \mathrm{~A}$-transcinnamate $4-$ monooxygenase $)$, COMT $=$ caffeic acid $O$-methyltransferase, $4 \mathrm{CL}=4$-coumarate-CoAligase, $\mathrm{HCT}=$ shikimate $O$-hydroxycinnamoyl transferase, $\mathrm{CCR}=$ cinnamoyl-CoA reductase, $\mathrm{CAD}=$ cinnamyl alcohol dehydrogenase, $\mathrm{POD}=$ peroxidase, $\mathrm{LAC}=$ laccases. Flavonoid pathway: $\mathrm{CHS}=$ chalcone synthase, $\mathrm{CHI}=$ chalcone isomerase, $\mathrm{F} 3 \mathrm{H}=\mathrm{flavanone}$ 3-hydroxylase, F3' H = flavonoid 3-hydroxylase, DFR = dihydroflavonol 4-reductase, LDOX = leucoanthocyanidin dioxygenase, FLS = flavonol synthase, LAR = leucoanthocyanidin reductase, ANR = anthocyanidin reductase, UFGT = UDP-glucose =flavonoid-3-O-glycosyltransferas. 
(Supplementary Figs. S16 and S17). An exception to this was observed for the DEGs with higher levels of expression at 3 versus $7 \mathrm{dpi}$, for which an increase in carbohydrate metabolic process annotations was observed.
Based on the findings from GO analyses and known virulence factors in fungi, we focused our detailed investigation on DEGs encoding proteins with molecular functions covering carbohydrate metabolism, transporters, TFs, kinases,
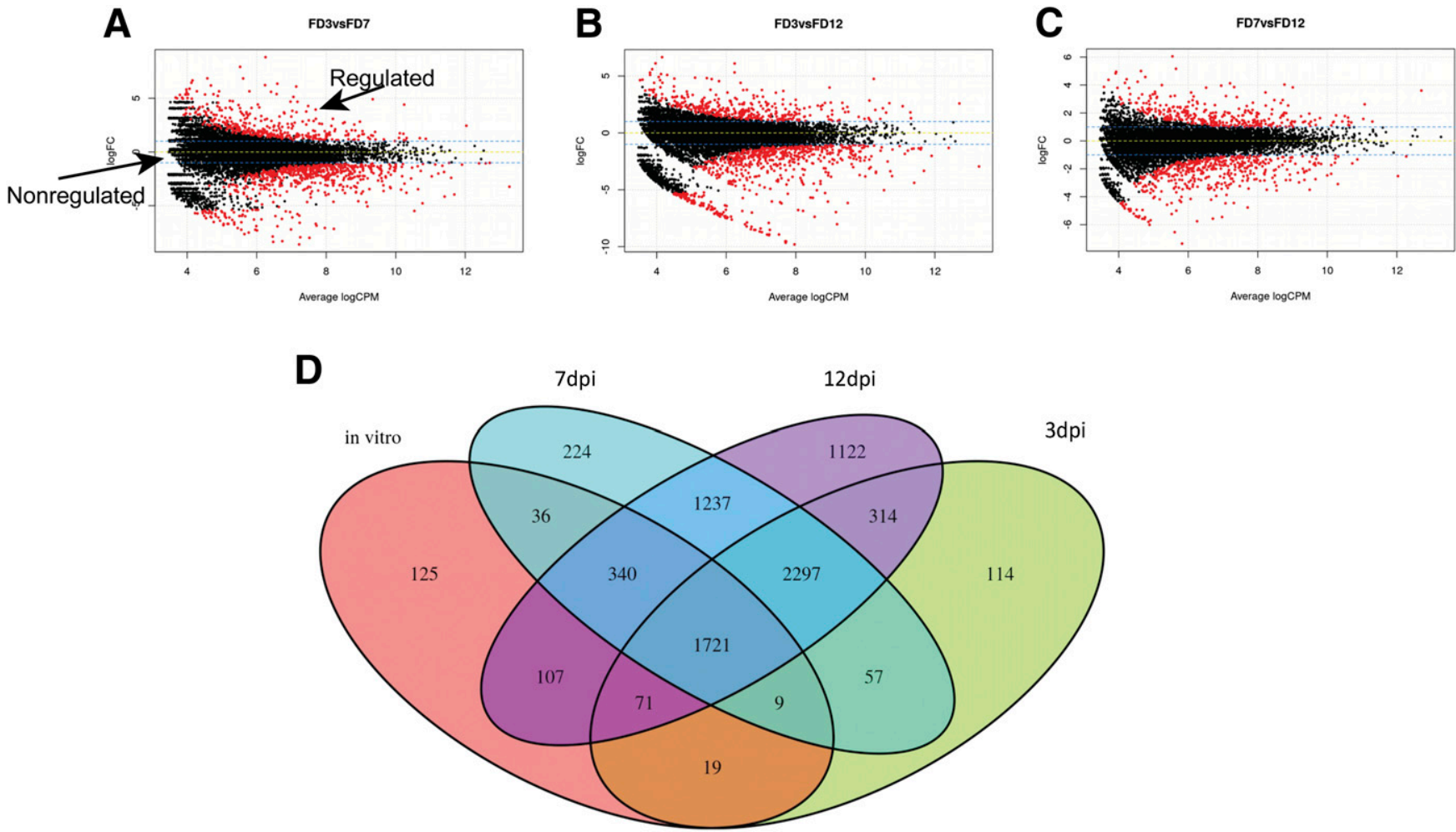

Fig. 6. Differentially expressed genes (DEGs) of Ramularia collo-cygni DK05 during infection of barley cv. Fairytale show the distribution of DEGs and nonregulated genes when comparing their expression level at $\mathbf{A}, 3$ versus 7 days postinfection (dpi), B, 3 versus 12 dpi, and $\mathbf{C}, 7$ versus 12 dpi. Each individual gene is plotted according to the fold change and the average expression (log counts per million). D, Venn diagram showing the number of $R$. collo-cygni expressed genes during in vitro and in planta growth at the analyzed time points.
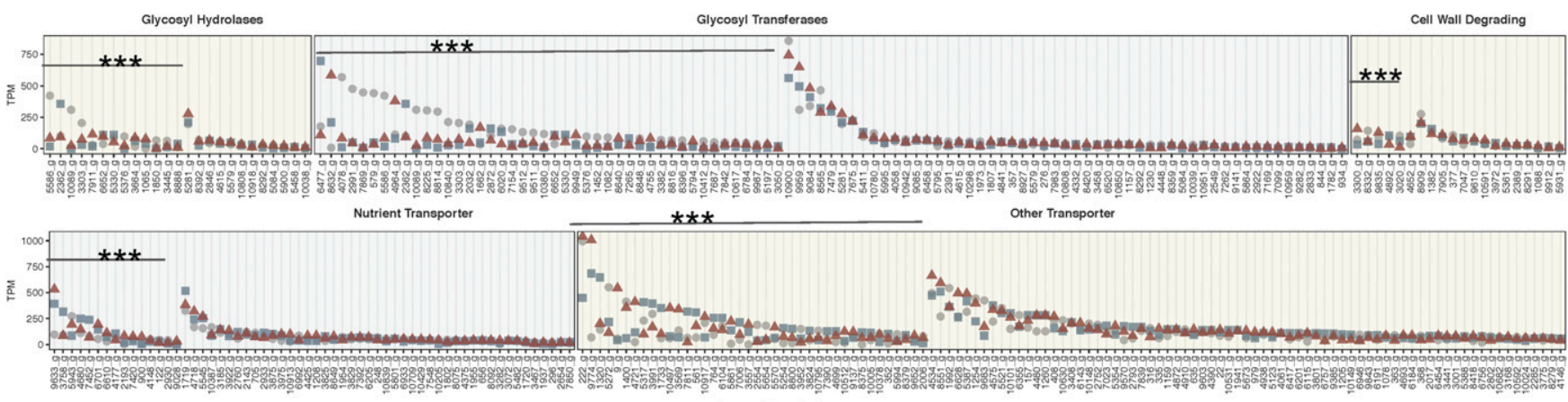

Transcription Factors

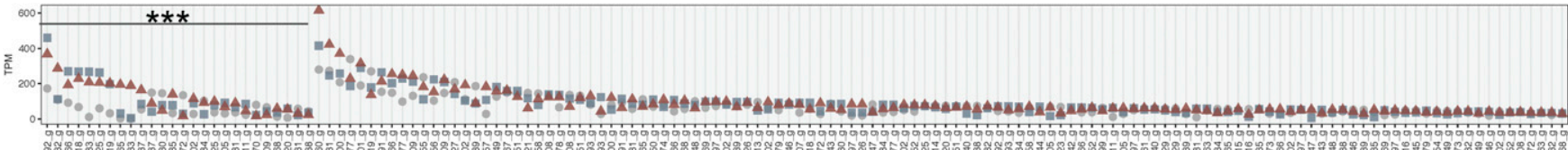

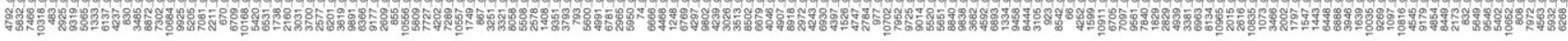

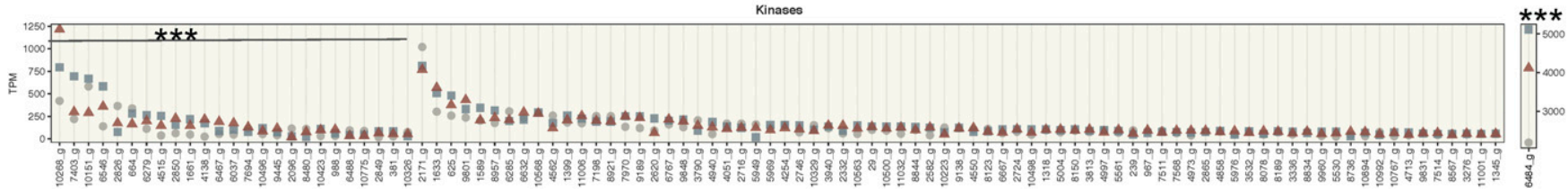

Fig. 7. Selected Ramularia collo-cygni genes robustly expressed (average transcripts per million [TPM] across all time points $>20$ ) or differentially regulated (***) during barley infection. The average TPM value at 3 (circle), 7 (square), and 12 (triangle) days postinoculation is shown. Genes are grouped on the basis of their predicted annotation. 
effectors, and toxins (Figs. 6 and 7) expressed during in planta colonization.

Mesophyll infection is accompanied by downregulation of $\mathrm{R}$. collo-cygni glycosyl hydrolases $(G H)$-coding genes. We identified $185 R$. collo-cygni genes with annotations covering carbohydrate metabolism, GH, glycosyl transferase, and cell wall-degrading enzymes (CWDE) that were expressed during barley infection. We found that 67 genes (Fig. 7) predicted to encode $\mathrm{GH}$ were expressed at all three time points. Two glucan 1,3- $\beta$-glucosidases, one endochitinase $\mathrm{A} 1$, and the cell-surface mannoprotein MP65 dominated the GH-annotated transcripts in planta (Supplementary Fig. S18). These had a high and relatively constant level of expression. Interestingly, 11 genes encoding $\mathrm{GH}$, predominantly xylanases and xylosidases, were found to be significantly downregulated in 3 versus 7-dpi and 3 versus 12-dpi comparisons. This indicates a rapid reprogramming of the expression of plant CWDE during infection. Three members of the GH32 family ( $\beta$-fructofuranosidase, levanase, exo-inulinase) and a trehalase were found to increase their expression significantly throughout the analyzed time points, indicating that metabolism of sugars is increased during in planta colonization. Among the 13 robustly expressed glycosyl transferases five were significantly regulated between 3,7 , and 12 dpi (Supplementary Fig. S19). A limited number of genes coding for CWDE is found in the $R$. collo-cygni genome (McGrann et al. 2016), and we found eight of these being robustly expressed in planta. Two of these CWDE were rapidly downregulated from 3 to $7 \mathrm{dpi}$.

R. collo-cygni expresses a large number of genes with predicted transporter function during barley infection. Analyses of the predominant functions expressed by R. collo-cygni during the interaction with $\mathrm{cv}$. Fairytale revealed expression of 307 genes predicted to encode transporters (Fig. 7). We investigated these to identify whether transport of specific nutrients or other small molecules was regulated through infection. We identified 168 genes encoding inorganic anion/cation transporters that were robustly expressed in planta, with homologs of the ammonium transporter MEP3, inorganic phosphate transporter PHO88, and siderophore iron transporter 1 having the highest levels of expression at all three time points (Supplementary Fig. S20). At 3 dpi, $R$. collo-cygni expressed, at a high level, genes involved in iron, metal-nicotianamine, phosphate, magnesium, and calcium transport. Expression of the ammonium transporter was increasingly dominant in the pool of transporter transcripts at 7 and $12 \mathrm{dpi}$. We found only a few anion/cation transporter genes significantly regulated between time points, with eight DEGs regulated at 3 versus 7 dpi or at 7 versus 12 dpi. Among these, we identified the ammonium transporter, calciumATPase2, copper, inorganic phosphate, manganese, and zinc transporters.

Analysis of the other classes of transporters identified 723 genes that were robustly expressed in planta, of which 44 and 59 were identified to be significantly regulated between 3 versus $7 \mathrm{dpi}$ or 7 versus $12 \mathrm{dpi}$, respectively. At $3 \mathrm{dpi}$, a major facilitator superfamily transporter, a peptide transporter similar to PTR2, and nucleotide and sugar transporters dominated these classes of transporters. This changed at 7 and $12 \mathrm{dpi}$, when the expression of the predicted efflux pump kojT and the aquaporins increased significantly (Supplementary Fig. S21).

R. collo-cygni expresses a limited number of TFs that are significantly regulated between the three time points. The $R$. collo-cygni genome contains 798 genes predicted to encode putative TFs, of which 238 were found expressed (TPM $>5$ ) in planta, but only 97 had a robust expression (average TPM >20) (Supplementary Fig. S22). The AN1-type zinc-finger protein TMC1 was found to have the highest expression level, followed by the atfl, FYVE-type zinc finger, hacl, and IIB TFs. Overall, only
16 genes predicted to encode TFs were found to be significantly regulated between 3 and $7 \mathrm{dpi}$ and 10 genes between 7 and $12 \mathrm{dpi}$.

Among the $245 R$. collo-cygni genes predicted to encode kinases, we identified 26 that were significantly regulated between 3 and $7 \mathrm{dpi}$ and five were identified between 7 and $12 \mathrm{dpi}$ (Supplementary Fig. S23). PEPCK has by far the highest expression levels at all time points (Fig. 7).

Predicted effector genes of $\mathrm{R}$. collo-cygni are actively regulated during barley infection. Effector proteins are crucial modulators of the interactions established by microbes with their compatible hosts. The $R$. collo-cygni genome contains 183 genes encoding for putative effector proteins identified by having a predicted signal peptide, no transmembrane region, and a cysteine content greater than $3 \%$. Among these, 17 were up-regulated and seven were down-regulated from 3 to $7 \mathrm{dpi}$, while 10 were up-regulated and 17 were down-regulated from 7 to 12 dpi (Fig. 8). Most of these predicted effectors ( $n=14$ at 3 versus $7 \mathrm{dpi}$ and $n=18$ at 7 versus $12 \mathrm{dpi}$ ) have no homolog in the Swissprot database (Supplementary Fig. S24), indicating no characterized protein domains, but similar proteins were identified in other fungal genomes, based on searches in the National Center for Biotechnology Information database. A LysM-containing protein and a predicted Ulo allergen protein were found to be significantly upregulated at 3 versus $7 \mathrm{dpi}$ and an intracellular hyphal protein 1 at 7 versus 12 dpi (Fig. 8B).

$\mathrm{R}$. collo-cygni modulates the production of SM at the transcriptional level. $R$. collo-cygni produces rubellin, a nonhost-specific, photodynamic anthraquinone toxin predicted to be derived from polyketides. Currently, the biosynthetic pathways of rubellin production and transport and secretion are unknown. Several genes involved in SM, including polyketide synthases, nonribosomal peptide synthases, and terpence cyclases or synthases were predicted in the $R$. collo-cygni genome and were previously shown to be expressed in planta (Dussart et al. 2018). A 40-kb region containing a possible metabolic gene cluster for rubellin biosynthesis was predicted based on comparative analyses of Aspergillus fulvum, Dothiostroma septosporum, and $R$. collocygni (McGrann et al. 2016). We analyzed the expression pattern of these genes during barley infection. Interestingly, we found that genes in the suggested metabolic cluster had very low levels of expression and no significant regulation across the three time points. If this cluster is involved in rubellin biosynthesis, it is likely not active in planta (Supplementary Fig. S25). By contrast, analysis of the expression level and pattern for other $R$. collocygni genes predicted to encode proteins involved in SM metabolism (aflatoxin, bikaverin, versicolorin, sterigmatocystin, or polyketides) identified several genes that were highly active or regulated in planta (Fig. 8C). Homologs of genes involved in versicolorin biosynthesis dominated the toxin-related transcriptome at all time points (Supplementary Fig. S26). Three genes predicted to code for versicolorin synthases were expressed in barley, but only two of them were significantly regulated between the analyzed time points and in an opposite direction. Two genes predicted to encode proteins similar to aflatoxin biosynthesis regulatory protein were expressed in planta. One was not expressed at 3 dpi but its transcript levels increased significantly and steadily at 7 and $12 \mathrm{dpi}$. The second gene had a low and constant expression pattern. Together these indicate that $R$. collo-cygni actively modulates SM production at the transcriptional level and that genes located in several clusters are likely to contribute to SM biosynthesis and transport.

\section{DISCUSSION}

$R$. collo-cygni infections of barley are often pathogenic with increasing negative effects on the final grain yield and quality 
(Havis et al. 2015b). To better understand this pathosystem, we set out to identify disease-associated molecular components in both the fungus and the host. We took advantage of the newly published genomes of $R$. collo-cygni and barley (Mascher et al. 2017; McGrann et al. 2016) and performed a detailed analysis of transcriptional changes associated with $R$. collo-cygni infection of barley based on simultaneous, unbiased genomewide transcriptional profiling of both organisms. A time- resolved RNA sequencing approach in a disease-promoting experimental setup based on infection of second leaves of barley seedlings was used. The pathogenic infection of $R$. collocygni on barley leaves was followed at three time points $(3,7$, and $12 \mathrm{dpi}$ ), corresponding to fungal entry through stomata, asymptomatic colonization of the mesophyll tissue, and formation of $R$. collo-cygni-induced RLS symptoms (Fig. 1). Mycelia-based inoculation of $R$. collo-cygni onto second-
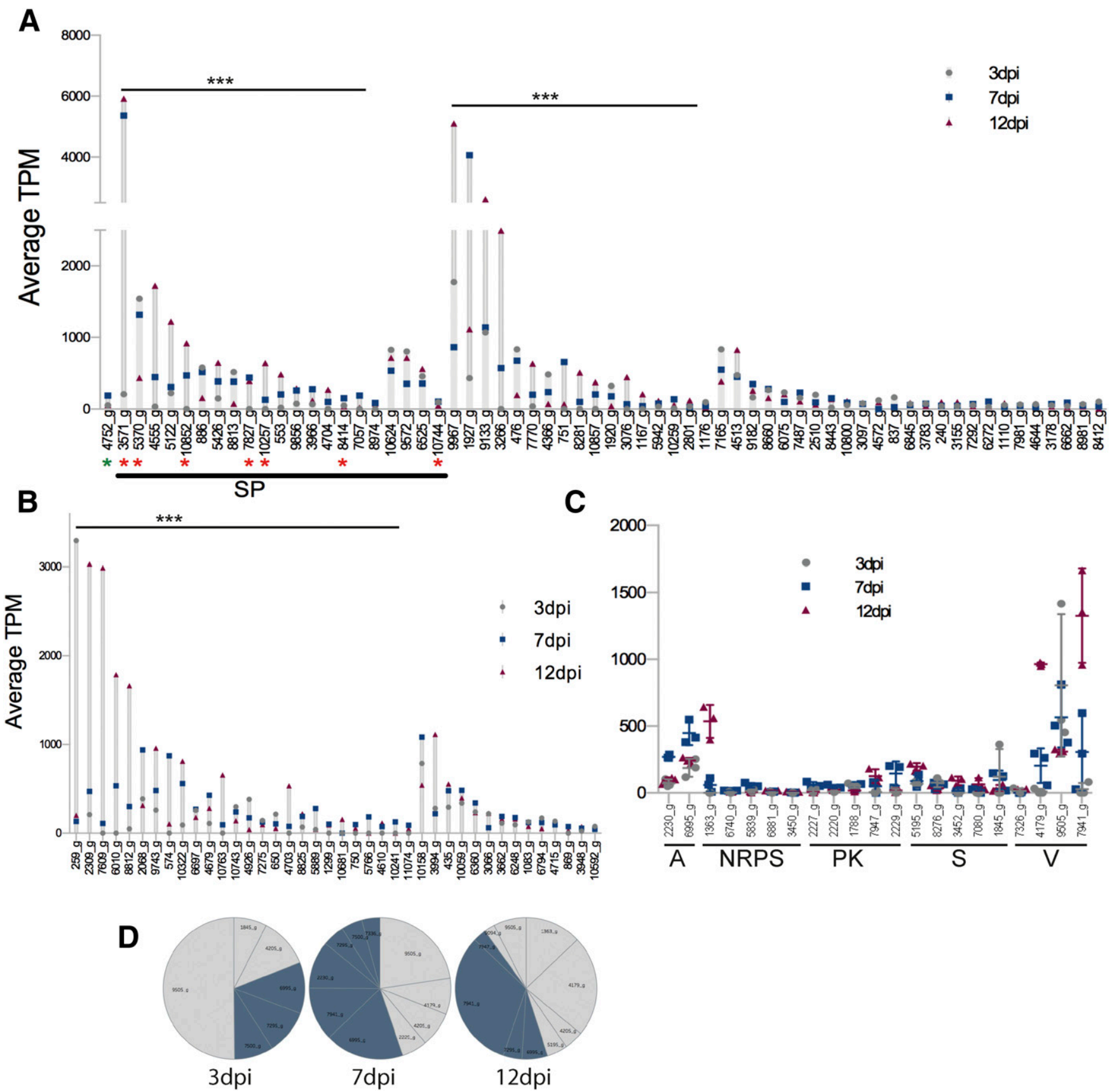

Fig. 8. Expression patterns at 3, 7, and 12 days postinfection (dpi) for genes predicted to encode Ramularia collo-cygni-specific proteins, effector proteins, or proteins involved in toxins metabolism. A, The average transcripts per million (TPM) values for genes that have no significant homolog in SWISSPROT and National Center for Biotechnology Information databases is shown. Genes are ordered first by the presence of a predicted signal peptide and transmembrane, signal peptide (SP), or no signal peptide, then by their significant regulation across any of the analyzed time points (three asterisks [***] indicate a false discovery rate $[\mathrm{FDR}]<0.05)$ and by the average TPM value $(>50)$ across all three time points. Genes marked with a single asterisk $(*)$ have a cysteine content $>3 \%$ and are, therefore, also considered as candidate effector proteins. B, The average TPM values of genes that are predicted to encode effector proteins (have a signal peptide and a cysteine content $>3 \%$, like those in A). Genes are ordered by their significant regulation across any of the analyzed time points (three asterisks [***] indicate FDR $<0.05)$ and by the average TPM values $(>50)$ across all three time points. $\mathbf{C}$, TPM values for genes predicted to encode proteins involved in toxin metabolism $(\mathrm{A}=$ aflatoxin, NRPS $=$ nonribosomal polyprotein synthesis, $\mathrm{PK}=$ polyketide, $\mathrm{S}=$ sterigmatocystin, $\mathrm{V}=$ versicolorin $)$ in the three biological replicates at 3, 7, and 12 dpi with the standard deviation (line). D, Expression level (average TPM) of genes involved in toxin biosynthesis (light) and toxin reduction (dark) as part of the overall expression of toxin-involvement genes at the three analyzed time points. 
formed barley leaves led to necrosis and chlorosis symptoms similar to RLS disease observed in the field on young plants and on upper canopy leaves after the flowering stage (Salamati and Reitan 2006; Walters et al. 2008). R. collo-cygni fungal biomass varied from 0.7 to $3.4 \%$ in the infected samples, and the proportion of $R$. collo-cygni-derived RNA reads reached from $0.15 \%$ and $1.3 \%$, comparable to previous findings from the Z. tritici-wheat pathosystem (Kellner et al. 2014; PalmaGuerrero et al. 2016; Rudd et al. 2015). In spite of the abundance of barley transcripts, the high sensitivity of RNA-seq approach permitted detection of expression of thousands of $R$. collo-cygni genes in the infected samples and identification of genes significantly regulated in the host and the fungus at different stages of infection. Analysis of the overall expression profiles revealed a subtle response of the barley transcriptome in the early infection and colonization stages ( 3 and $7 \mathrm{dpi}$ ). Transcription of $R$. collo-cygni genes was dynamic through the different infection stages. Based on these findings from $R$. collocygni and barley transcriptional regulation, we developed a narrative model of the molecular events associated with RLS disease development in barley (Fig. 9).

\section{Transcriptional changes accompanying $R$. collo-cygni establishment on barley leaves ( $3 \mathrm{dpi}$ ).}

At $3 \mathrm{dpi}$, in our experimental set-up, $R$. collo-cygni was predominantly present as growing mycelia on the surface of barley leaves, with only a few, sporadic stomatal entries (Fig. 1). In spite of this very limited infection and in the absence of phenotypic responses in the host, we found a pronounced reaction at the transcriptional level. A large proportion $(69 \%)$ of $R$. collo-cygni genes were expressed at this early stage, many at higher levels than at 7 or 12 dpi while, in barley, nearly 500 transcripts were significantly regulated compared with the untreated condition. Together, these demonstrate that microbial recognition had occurred and that the two partners had initiated molecular responses by 3 dpi. On the fungal side, we identified genes encoding pathogenicity factors, some with potential hallmarks of secreted effectors, being highly expressed at $3 \mathrm{dpi}$, including a chitin-binding LysM protein. In response to the fungus, the expression of several RLKs was induced in the barley host, among which was an ortholog of the rice chitin elicitor receptor kinase CERK1. This likely represents classical chitin-elicited responses described for fungal-plant pathosystems (Sánchez-Vallet et al. 2015). Many R. collo-cygni genes encoding proteins that may target the barley cell wall (xylanase, glucanase, pectate lyase, esterase, and cutinase) or may degrade host proteins (proteases) were found highly expressed at $3 \mathrm{dpi}$. These were accompanied by upregulation of chitinases and glucan endo-1,3- $\beta$-glucosidases in barley, indicating host attack of fungal cell-wall components. Many transporters, especially those with anion/cation transport annotation and those transporting unknown metabolites were highly expressed by $R$. collocygni at this early stage, indicating a need for import/export processes to support mycelial growth. Several TFs are expressed by $R$. collo-cygni or induced in barley likely to coordinate these transcriptional responses. In barley, these include WRKY and NAC TFs, known to activate transcripts encoding PR and woundinduced proteins (Christianson et al. 2010; Eulgem 2006). Congruent with this, we found several genes encoding defense proteins to be strongly induced (Fig. 4), implying that barley recognizes $R$. collo-cygni PAMPs and breakdown products from the activity of $R$. collo-cygni secreted CWDE and activates basal defense. Induced expression of several genes encoding peroxidases indicates that formation of ROS may potentiate this defense by acting as executioners of $R$. collo-cygni in the apoplast or by further defense-gene activation. Another prominent host response at 3 dpi was strong induction of SM transporters together with phenylpropanoid and flavonoid pathway genes, suggesting that the immediate defense strategy also involves production and secretion of plant phytotoxic compounds. An overall downregulation of photosynthesis-related gene expression has previously been suggested as an adaptive response to biotic attack (Bilgin et al. 2010). Both MAMP recognition and ETI activation of the mitogen-activated protein kinases MPK3 and MPK6 confer rapid transcriptional reprogramming of chloroplast encoded transcripts, global downregulation of photosynthesis, along with upregulation of defense-related genes and signaling pathways (de Torres Zabala et al. 2015; Ishiga et al. 2017; Su et al. 2018). The mechanism is suggested to involve induction of stromules to connect chloroplasts with the nucleus and possibly use them as a signaling bridge guiding chloroplast-generated ROS into

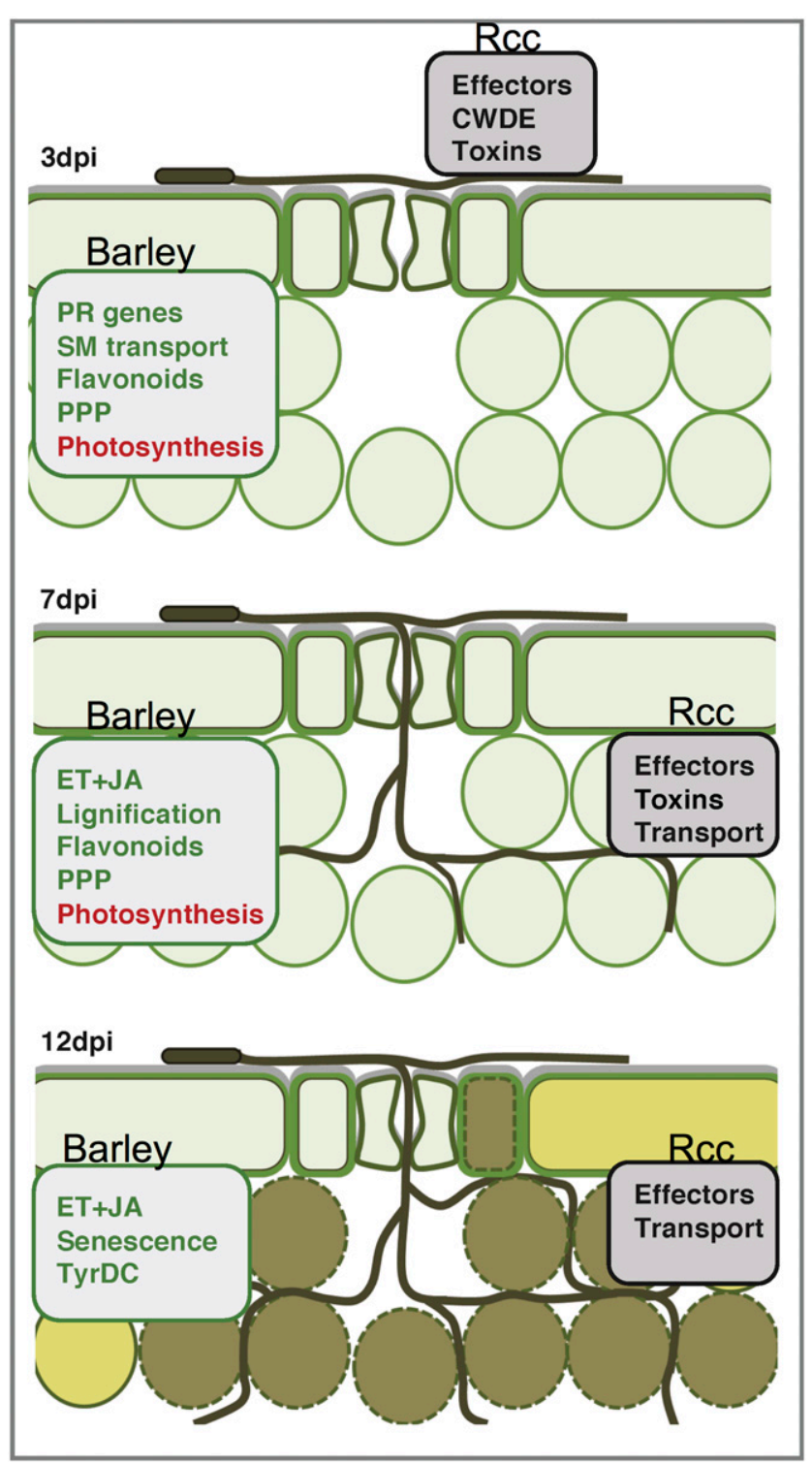

Fig. 9. Schematic representation of Ramularia collo-cygni (Rcc) infection of barley and the associated main molecular components identified to be induced or downregulated at the three time points $(3,7$, and 12 days postinfection [dpi]) in $R$. collo-cygni and barley. At 3 dpi, there is fungal growth on leaf surface and barley cells are alive; at $7 \mathrm{dpi}$, there is hyphal growth in the mesophyll and barley cells are alive; at $12 \mathrm{dpi}$, there is hyphal growth in the mesophyll and barley chlorosis and cell death is observed. CWDE $=$ cell wall-degrading enzymes, $\mathrm{PR}=$ pathogenesis-related genes, $\mathrm{SM}=$ secondary metabolites, $\mathrm{PPP}=$ phenylpropanoid pathway, $\mathrm{ET}=$ ethylene signaling, $\mathrm{JA}=$ jasmonic acid signaling, and $\mathrm{TyrDC}=$ tyrosine decarboxylase. 
the nucleus to promote transcriptional reprogramming (Caplan et al. 2015; Kumar 2018). In our study, we observed a strong downregulation of genes encoding early light-induced proteins from the beginning of $R$. collo-cygni infection, followed by a subtle down regulation of the whole photosynthesis system throughout $R$. collo-cygni colonization. We envision this downregulation as representing part of the immune response in barley and an attempt by the host to protect itself from unwanted oxidative stress from photosynthesis that might add to the overall ROS challenge experienced by the cells during attack and defense against $R$. collo-cygni.

\section{Barley and $R$. collo-cygni transcriptional responses associated with the asymptomatic colonization of the leaf mesophyll ( $7 \mathrm{dpi})$.}

At $7 \mathrm{dpi}$, we found $R$. collo-cygni infecting the mesophyll apoplast (Fig. 1E and F). The infection was still asymptomatic, as no macroscopic signs of disease were observed on the leaves (Fig. 1A). Mesophyll infection was accompanied by major and diverse changes in $R$. collo-cygni and barley transcriptomes. Host manipulation remained a theme, as $R$. collo-cygni expressed several genes encoding putative effector proteins, some of which are currently $R$. collo-cygni-specific (Fig. 8B). Apoplast colonization was found associated with reduction in the expression of $R$. collo-cygni genes predicted as GH and CWDE. This could represent a fungal response to transcriptional activation of xylanase inhibitors and invertase/pectin methylesterase inhibitor superfamily proteins by the barley host, probably to protect against $R$. collo-cygni CWDE. In parallel, expression of enzymes important for reinforcement of host cell walls by lignification (e.g., monolignols), initiated at $3 \mathrm{dpi}$, was increased at $7 \mathrm{dpi}$. Phenylpropanoid-derived lignin acts by cross-linking plant secondary cell walls to provide mechanical strength for defense (Vanholme et al. 2010). We identified strong activation of lignin biosynthesis genes during asymptomatic $R$. collo-cygni colonization, with caffeic acid $O$ methyltransferase and 4-coumarate-CoA ligase genes being highly expressed (Fig. 5B). Beside lignin formation, barley also seems to utilize phenolic compounds to form flavonoids. Many flavonoids act as phytoalexins with antifungal properties and are produced in response to pathogen attack (Treutter 2005). A strong increase in the expression of barley genes encoding key flavonoid pathway enzymes was observed from 7 dpi in $R$. collo-cygni-inoculated leaves (Fig. 5C). These likely reflect an attempt to control $R$. collo-cygni colonization by production of flavonoid phytoalexins. Based on the upregulation of $R$. collo-cygni trihydrophobins, heat-shock proteins, and genes involved in oxido-reductive processes, it is likely that colonization of barley apoplast induces water surface tension on the fungal hyphae and increased host-imposed stresses. Upregulation of a formate transporter and formate dehydrogenase, together with the induction of nitrate reductase, could indicate a possible anaerobic metabolism in $R$. collo-cygni during apoplastic colonization. We interpret the upregulation of ammonium transporter MEP3 from $7 \mathrm{dpi}$ as either an active ammonium fermentation, as observed for Fusarium oxysporum (Zhou et al. 2002), or as an increased uptake of ammonium from the apoplast to support accelerated metabolic activities in the invading fungus. Future biochemical characterization of the properties of $R$. collo-cygni MEP3 may be relevant for understanding the life style of $R$. collo-cygni during barley mesophyll infection prior to disease symptom development. The strong induction of genes involved in the uptake and metabolism of hexoses at $7 \mathrm{dpi}$ (Supplementary Fig. S27) indicate that $R$. collo-cygni is most likely acquiring necessary carbon resources from the apoplast. Activation of the glyoxylate cycle could indicate the use of internal or host-derived fatty acids, as previously shown to be important for pathogenic fungi (Lee et al. 2009; Lorenz and Fink 2001). Hexose feeding could also explain the reduction in $R$. collo-cygni cutinase transcript levels at this stage, as cutinase expression was shown to be suppressed by glucose (Bajar et al. 1991). Sensing $R$. collocygni presence in the apoplast and, possibly, its nutrientdepleting activities, is likely to involve $\mathrm{Ca}^{2+}$ as a second messenger to trigger defense responses in barley cells. Calmodulins (CaM) and calmodulin-like (CML) genes act as calcium-sensor proteins and orchestrate cellular signaling cascades toward defense responses against pathogenic attack (Zhang et al. 2014). A total of 24 barley transcripts of CaM, CML, or CaM-binding proteins were strongly up-regulated at $7 \mathrm{dpi}$, indicating strong activation of calcium signaling. Barley MLO, a seven transmembrane-domain protein, interacts with $\mathrm{CaM}$ and negatively regulates defense responses, thereby promoting susceptibility to powdery mildew fungi (Büschges et al. 1997; Consonni et al. 2006; Kim et al. 2002). Lack-of-function mlo mutations lead to effective powdery mildew disease resistance, but enhanced susceptibility to several facultative pathogens (Jarosch et al. 1999; Jansen et al. 2005; Kumar et al. 2001). Previous studies suggested that the widespread use of mlo resistance in barley cultivars with the purpose of controlling powdery mildew disease may have stimulated the emergence of RLS as a major barley disease (Makepeace et al. 2007; McGrann et al. 2014). The barley cultivar Fairytale, used in this study, contains the wild-type Mlo gene, and we found a limited (1.3-fold) but significant increase in its expression in the $R$. collo-cygni-inoculated leaves at $7 \mathrm{dpi}$ (Fig. 4). We speculate that increased Mlo expression could mediate defense against $R$. collo-cygni, but that $R$. collo-cygni may use effectors to effectively suppress Mlo (Lewis et al. 2012) and, consequently, increase barley susceptibility.

A prominent response of barley to $R$. collo-cygni infection at 7 dpi was observed in phytohormone biosynthesis and signaling pathways (Fig. 5A). It is widely accepted that plant defense against necrotrophic pathogens is mediated by JA and ET pathways, whereas, in response to an attack of biotrophic pathogens, the plant employs SA signaling (Grant and Jones 2009; McGrann et al. 2014). The infection strategy of $R$. collocygni is still poorly understood, though it is thought to change from an endophytic lifestyle during the early barley-growing season to a pathogenic, symptomatic phase late in the season. Only a few genes associated with phytohormone pathways were activated already at $3 \mathrm{dpi}$. Interestingly, these were related to biosynthesis of JA and ET, and even though we observed a strong activation of PR1 transcripts, no regulation of expression of genes belonging to the SA pathways was found. This suggests that $R$. collo-cygni infection activates the ET and JA pathways simultaneously. This response was observed to build over time through the asymptomatic colonization by $R$. collocygni (7 dpi) and during development of RLS disease symptoms (12 dpi) (Figs. 5A and 9). Increased JA and ET production may be responsible for the observed activation of ETresponsive TF-like proteins. These, in turn, may activate PR genes, including genes encoding thaumatin-like proteins that have antifungal activity (Liu et al. 2010), possibly to prevent further progress of $R$. collo-cygni infection.

\section{Transcriptome responses at the stage of RLS symptom development (12 dpi).}

The $R$. collo-cygni-inoculated barley leaves showed typical RLS symptoms at 12 dpi, i.e., necrotic spots surrounded by a yellow chlorotic halo. RLS symptoms have been associated with the ability of $R$. collo-cygni to produce anthraquinone toxins called rubellins, which induce necrotic lesions similar to those observed during fungal infection (Heiser et al. 2003). 
Consequently, it has been suggested that rubellins are the cause of the infected leaves (Miethbauer et al. 2003). A specific cluster of genes in the $R$. collo-cygni genome was predicted to encode proteins involved in rubellin production, based on its similarity to the aflatoxin cluster from Aspergillus spp. (McGrann et al. 2016). In our in-planta transcriptome data, these genes were not found to be expressed, suggesting that either rubellin is not produced by $R$. collo-cygni during barley leaf colonization or that this gene cluster is not responsible for rubellin production. A more recent study found that $R$. collocygni expresses numerous genes required for the production of a diverse panel of SM, some with antimicrobial activity (Dussart et al. 2018). Our analysis, based on in-planta RNAseq, revealed a similar situation in which the expression of several genes encoding enzymes predicted to be involved in the metabolism of various SM were highly modulated according to the infection stage. A preponderance of those involved in SM biosynthesis with higher levels of expression at both 3 and 7 dpi were found (Fig. 8D). Toxin production, in general, requires a large pool of electrons, and rubellin biosynthesis or activity was shown to be promoted by acidic conditions (Miethbauer et al. 2003). We therefore inspected the $R$. collo-cygni transcriptome data for possible molecular signatures of toxin promoting metabolic activities. We found increased expression of formate transporter and formate dehydrogenase. These could contribute to the pool of electrons necessary for cytochrome P450s, which also have higher levels of expression in planta and enable the required energy for driving toxin production. Additionally, the $\mathrm{CO}_{2}$ resulting from formate dehydrogenase activity might be taken up by the upregulated carbonic anhydrase to produce bicarbonate and protons and create an acidic environment that promotes rubellin formation or activity. Interestingly, we found that a large proportion of genes involved in SM detoxification had an increased expression at 12 dpi (Fig. 8D). This might act to reduce the toxic effects of SM on $R$. collo-cygni, enabling fungal growth and survival. However, the exact role of SMs during $R$. collo-cygni infection and their potential involvement in RLS disease symptom formation remains speculative at this stage.

Rubellin induces light- and concentration-dependent necrosis on barley leaves, probably by triggering light-dependent production of ROS (Heiser et al. 2003). Ascorbate and glutathione are key players in controlling ROS (Noctor and Foyer 1998), and the induction of several genes encoding these enzymes at the time of RLS formation suggest that they could be involved in containment and protection against rubellintriggered ROS in infected leaves. Rubellin was also shown to induce fatty acid peroxidation, which, in turn, was shown to be associated with natural leaf senescence in barley (Heiser et al. 2004). We explored the regulation pattern of barley genes associated with senescence in our infection experiment. In the control samples, leaves appeared healthy and green with no visible signs of senescence during the investigated time points. Congruently, we observed no significant changes in the expression pattern of senescence-associated TFs, such as S40, NAC5, and WRKY12, or other senescence-associated genes, such as Autophagy gene 8, Papain-type cysteine peptidase, and Pheophorbide A oxygenase, involved in senescence-associated remobilization and degradation of nutrient resources (Kucharewicz et al. 2017). This indicates that natural senescence was not induced in uninfected barley leaves in the time frame of our experiment. However, in the $R$. collo-cygni infected leaves, these senescence-associated genes were upregulated, resembling patterns observed in naturally senescing barley leaves (Kucharewicz et al. 2017). This suggest that, as has been observed for wheat during Z. tritici infection (Ma et al. 2018), senescence processes are induced in barley leaves during
R. collo-cygni infection and contribute to formation of RLS disease symptoms, however, nutrient resources might not be host-available but, rather, $R$. collo-cygni-exploited. Increased expression of barley transmembrane sugar transporters was observed at this stage of $R$. collo-cygni colonization and transport of sugars, probably to the apoplast, may further promote $R$. collo-cygni growth. $R$. collo-cygni, in turn, upregulated its own sugar and nutrient transporters. At this later stage of the infection, we found that cellular signaling for defense was more intense. Among the most highly $R$. collo-cygni-induced transcripts were several barley genes annotated as tyrosine decarboxylases (TyrDC). Aromatic 1-amino acid decarboxylases are suggested to play crucial roles in the biosynthesis of a number of different SM in plants, and TyrDC may be involved in plant defense. Both TyrDC transcript level and TyrDC activity transiently respond to fungal elicitor treatment in Arabidopsis thaliana cell-suspension cultures (Trezzini et al. 1993). In barley, TyrDC is involved in the biosynthesis of hordenine (Leete and Marion 1953), an alkaloid that is toxic to fungi (Lovett and Hoult 1995) that may be produced by the host in the attempt to provide protection against $R$. collo-cygni. However, hordenine expression has been found only in young barley roots (Mann and Mudd 1963), and therefore, it remains to be established if or to what extent hordenine might be produced in the barley leaves during $R$. collo-cygni infection.

In conclusion, our data shows that $R$. collo-cygni displays molecular signatures of a pathogenic fungus while infecting barley leaves and triggers defense responses in the plant host already during the asymptomatic stages. However, in the analyzed cultivar, the initiated defense did not prevent $R$. collocygni colonization and development of RLS disease symptoms. It is likely that expression of diverse $R$. collo-cygni virulence factors during leaf infection may contribute collectively to RLS disease symptoms. Identifying their role for $R$. collo-cygni pathogenicity in various barley cultivars will be important for better understanding and prevention of RLS disease.

\section{MATERIALS AND METHODS}

\section{Sample preparation.}

Plant and fungal growth conditions. Barley (Hordeum vulgare) cv. Fairytale was grown under a long-light period ( $16 \mathrm{~h}$ of light, $300 \mu \mathrm{mol} \mathrm{m} \mathrm{m}^{-1}$ and $8 \mathrm{~h}$ of darkness) at constant $19^{\circ} \mathrm{C}$. Fourteen-day-old plants were used for inoculation.

Ramularia collo-cygni DK05 was grown in the dark on potato dextrose agar plates supplemented with streptomycin $(10 \mu \mathrm{g} / \mathrm{ml})$. Liquid cultures were prepared 3 weeks before inoculation by transferring three agar plugs into $75 \mathrm{ml}$ of potato dextrose broth media supplemented with streptomycin $(10 \mu \mathrm{g} / \mathrm{ml})$. Cultures were grown at room temperature in the dark and under constant agitation at $120 \mathrm{rpm}$ until use.

Fungal inoculation. Second-formed leaves were fixed in horizontal position with the adaxial side upwards, using cotton strings and a plastic support. Fungal hyphae were collected after filtration through a cotton cloth, were washed with $500 \mathrm{ml}$ of double-distilled water, and were suspended in $15 \mathrm{ml}$ of water. Hyphae were fragmented by sonication (three times for $30 \mathrm{~s}$ each). Suspensions were rested for 2 min to allow larger hyphal fragments to settle, before the supernatant was collected. The hyphal concentration was adjusted to $5 \times 10^{5}$ hyphae per milliliter and Tween 20 (at $4 \mu \mathrm{l} / \mathrm{ml}$ ) was added. Leaves were sprayinoculated with the mycelial suspension, using an atomizer (Desaga, Sarstedt-Gruppe) until run-off. After inoculation, plants were placed under plastic covers to maintain high relative humidity (80 to $100 \%$ ) and were kept for $48 \mathrm{~h}$ in darkness. After this incubation time, plants were maintained in the normal 16-h day and 8-h night rhythm. Noninoculated control 
plants were inoculated with water and Tween $20(4 \mu \mathrm{l} / \mathrm{ml})$ and were maintained under the same conditions as the inoculated plants. Leaves were inspected visually on a daily basis, and samples were collected at 3, 7, and 12 dpi. For fungal biomass quantification and RNA sequencing, leaf segments from three different leaves were pooled into one sample. For microscopy, three leaf segments were cleared and fixed as previously described (Lyngkjaer et al. 1997). The experiment was repeated three times, using independently grown cultures of $R$. collocygni inoculum.

DNA and RNA extractions. Leaf samples were snap-frozen in liquid nitrogen. RNA and DNA were extracted from the same sample after a modified protocol from Yang et al. (2008). Samples were ground using a tissue-lyser for 2 min and were then incubated for $10 \mathrm{~min}$ at $65^{\circ} \mathrm{C}$ with $900 \mu \mathrm{l}$ of extraction buffer (2\% CTAB, 0.1 M Tris-HCL [pH 8], 1.4 M NaCl [5 M], $20 \mathrm{mM}$ EDTA [pH 8], 2\% polyvinylpolypyrrolidone) and $100 \mu \mathrm{l}$ of $\beta$-mercaptoethanol. Tubes were briefly shaken every $2 \mathrm{~min}$ during incubation. Subsequently, $1 \mathrm{ml}$ of chloroform was added, and samples were vortexed to form an emulsion, then centrifuged for $20 \mathrm{~min}$ at $4^{\circ} \mathrm{C}$ at $10,000 \times g$. An equal volume of phenol/chloroform/isoamylalcohol $(25: 24: 1)$ was added to the supernatant in a new tube and was mixed thoroughly. Centrifugation was performed as before. The top aqueous layers were mixed with 1 volume of chloroform/isoamylalcohol (24:1) and were centrifuged as before. One-third volume of $\mathrm{LiCl}(8 \mathrm{M})$ was added to the supernatant and was incubated overnight at $-20^{\circ} \mathrm{C}$. Samples were centrifuged for $30 \mathrm{~min}$ at $4^{\circ} \mathrm{C}$ and $10,000 \times g$. The RNA pellet was washed twice with $70 \% \mathrm{EtOH}$ and was air-dried. The supernatant was used for DNA precipitation by adding two volumes of $96 \% \mathrm{EtOH}$ and one-tenth volume of $\mathrm{NaCl}(5 \mathrm{M})$ and incubating for $20 \mathrm{~min}$ at $-20^{\circ} \mathrm{C}$, followed by centrifugation for $30 \mathrm{~min}$. The DNA pellet was washed with $70 \% \mathrm{EtOH}$. DNA and RNA were resuspended in $50 \mu \mathrm{l}$ double distilled $\mathrm{H}_{2} \mathrm{O}$.

Fungal biomass measurement. Primers for detection of $R$. collocygni were designed to amplify a 174-bp fragment specific to Ramularia species from the internal transcribed spacer (ITS) region, GenBank KJ504786.1. The primers used were as follows:

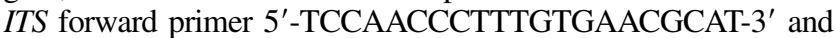
reverse primer $5^{\prime}$-ATTTCGCTGCGTTCTTCATCGA-3'. To normalize the amount of fungal DNA in the leaves to plant DNA, the elongation factor gene $(E F O)$ from barley was used, with $E F O$ forward primer 5'-ACCCTGACAAGGTTCCCT TC-3' and reverse primer $5^{\prime}$-ACCAGTCAAGGTTGGTGG AC- $3^{\prime}$. The quantitative PCR was performed with the BioRad CFX384 real-time PCR detection system, using the KAPA SYBR Fast qPCR mix.

RNA-seq analysis of infected and uninfected samples. Library preparation and sequencing were performed by Edinburgh Genomics. Libraries were prepared using the TruSeq stranded mRNA-seq kit from Illumina and were sequenced on an Illumina HiSeq4000 (150 bases paired end). Each sample was split over six lanes. A FastQC report was made for each fastq file. The reports were summarized using MultiQC and were visually inspected. Raw data were trimmed of adapters and low-quality base calls using Skewer. Raw data have been deposited in the ENA read archive under BioProject PRJEB14791. The data from each lane was analyzed separately as well as merged by sample, to the point of data integrity control through multi-dimensional scaling (MDS) plots. After this, the data were analyzed by sample only.

Reannotation of the R. collo-cygni genome, RNA-seq read mapping, and read counting. The previously published gene and protein files (McGrann et al. 2016) did not provide complete gene models for all $R$. collo-cygni genes. We therefore took advantage of our new
RNA seq dataset to perform an improved gene annotation. The RNA seq data were mapped to the published genome using Braker (Hoff et al. 2016), which utilizes both GeneMark-ET (Lomsadze et al. 2014) and Augustus. The GeneMark-ET annotation was subsequently used, in this study, because of its optimality for fungal gene annotation (Hoff et al. 2016). The new R. collocygni gene models were functionally annotated using several tools and databases (Supplementary Fig. S1). To identify Pfam domains, GO annotations, signal peptides and transmembrane peptides, Interproscan (Jones et al. 2014; Mulder and Apweiler 2007) was used. To find glycosylphosphatidylinositol (GPI)anchored proteins a GPI-anchor positive dataset and a GPI-anchor negative dataset were downloaded (GPI-anchor Predictor website) and predicted proteins were compared with these using BLAST (Altschul et al. 1990). Predicted proteins were also compared using BLAST to the PHI database to find homologs with verified pathogenicity. WoLF PSORT (Horton et al. 2007) was used to predict protein localization.

Competitive mapping was performed to separate the RNA reads derived from the host and fungus. The genomes of R. collo-cygni (McGrann et al. 2016) and barley (Mascher et al. 2017) were merged to one file using samtools merge ( $\mathrm{Li}$ et al. 2009), and the published high confidence gene prediction of the barley and our new predictions for $R$. collo-cygni were merged into one gtf file. STAR (Dobin et al. 2013) was used to map the trimmed reads to the merged genomes, using the two-pass approach for accurate splice annotation. Mapping quality and sequencing depth was assessed with RSeQC (Wang et al. 2012) and was collated with MultiQC (Ewels et al. 2016). Reads were counted using HT-seq (Anders et al. 2015), which performs a per-gene base counting where a read pair overlaps an exon, in part or in full. After read counting per sample, the counts were separated based on species.

TPM calculation, differential gene expression analyses, and functional interpretation. For a second assessment of expression, accurate assessment of genes belonging to gene families, isoform detection, and a gene expression estimate for comparability to other studies, TPM values were calculated using RSEM (Li and Dewey 2011). RSEM is a transcript-based estimator that recalculates the probability of a read mapping to a transcript using a Bayesian model. This makes it possible to compare the expression values of the in-planta fungal transcripts to the previously published in-vitro transcript data (McGrann et al. 2016). To accurately calculate the fractions per library for each species, the reads had to be split by species before remapping and counting. For this reason, bam files from competitive mapping were first split by contig, merged based on sample and species, and then, the reads, which were properly paired, were extracted from each bam file using Samtools ( $\mathrm{Li}$ et al. 2009). The reads were subsequently mapped to the respective genome using Star (Dobin et al. 2013), and RSEM was run independently on barley and $R$. collo-cygni bam files. The previously published transcriptome of $R$. collo-cygni (McGrann et al. 2016) was also mapped to the $R$. collo-cygni genome using Star, and TPM values were calculated using RSEM. A Trinity script (Haas et al. 2013) using the trimmed mean method (TMM) was applied to normalize TPM values between samples.

The R package EdgeR (Robinson et al. 2010) was used to perform MDS analysis as well as differential expression analyses based on the HT-seq counts for each species separately. For each of the two datasets, the samples were normalized using the inbuilt TMM method. Differential gene expression was performed according to the GLM method, in which the three independent biological replicas of our experiment were considered. In our downstream analyses, we have included barley genes that were differentially expressed (DEGs) $\left(\log _{2}\right.$ 
FC $>1$, false discovery rate $<0.05$ ) compared with control, uninoculated samples at the corresponding time point and had a TMM value of 10 in at least three samples. For $R$. collo-cygni, a minimum TPM value of 5 in all biological replica was used as a criterion for 'expression', and an average TPM value of 20 across all time points for 'robust expression' in planta. Tableau v.10.4 software was used for data visualization.

GO enrichment analyses were performed on the DEGs in barley, using GOATools. A generic GO-slim file was downloaded from the Gene Ontology Consortium website and was used for creating a GO-association file from the barley GO annotations with a consistent hierarchy and resolution. This file was used for $\mathrm{GO}$ enrichment analyses together with each list of up- or downregulated DEGs.

\section{ACKNOWLEDGMENTS}

We thank R. L. Hjortshøj (Sejet PlantBreeding, Aps, Denmark) for seed material and constructive discussions, J.-B. Lopez for constructive discussions, and T. Yarra for helpful discussions on the statistical analyses.

\section{LITERATURE CITED}

Acevedo-Garcia, J., Kusch, S., and Panstruga, R. 2014. Magical mystery tour: MLO proteins in plant immunity and beyond. New Phytol. 204: 273-281.

Altschul, S. F., Gish, W., Miller, W., Myers, E. W., and Lipman, D. J. 1990 Basic local alignment search tool. J. Mol. Biol. 215:403-410.

Anders, S., Pyl, P. T., and Huber, W. 2015. HTSeq-A Python framework to work with high-throughput sequencing data. Bioinformatics 31:166-169.

Bajar, A., Podila, G. K., and Kolattukudy, P. E. 1991. Identification of a fungal cutinase promoter that is inducible by a plant signal via a phosphorylated trans-acting factor. Proc. Natl. Acad. Sci. U.S.A. 88: 8208-8212.

Bigeard, J., Colcombet, J., and Hirt, H. 2015. Signaling mechanisms in pattern-triggered immunity (PTI). Mol. Plant 8:521-539.

Bilgin, D. D., Zavala, J. A., Zhu, J., Clough, S. J., Ort, D. R., and DeLucia E. H. 2010. Biotic stress globally downregulates photosynthesis genes. Plant Cell Environ. 33:1597-1613.

Büschges, R., Hollricher, K., Panstruga, R., Simons, G., Wolter, M., Frijters, A., van Daelen, R., van der Lee, T., Diergaarde, P., Groenendijk, J., Töpsch, S., Vos, P., Salamini, F., and Schulze-Lefert, P. 1997. The barley Mlo gene: A novel control element of plant pathogen resistance. Cell 88:695-705.

Caplan, J. L., Kumar, A. S., Park, E., Padmanabhan, M. S., Hoban, K., Modla, S., Czymmek, K., and Dinesh-Kumar, S. P. 2015. Chloroplast stromules function during innate immunity. Dev. Cell 34:45-57.

Choi, H. W., and Klessig, D. F. 2016. DAMPs, MAMPs, and NAMPs in plant innate immunity. BMC Plant Biol. 16:232

Christianson, J. A., Dennis, E. S., Llewellyn, D. J., and Wilson, I. W. 2010 ATAF NAC transcription factors: Regulators of plant stress signaling. Plant Signal. Behav. 5:428-432.

Consonni, C., Humphry, M. E., Hartmann, H. A., Livaja, M., Durner, J., Westphal, L., Vogel, J., Lipka, V., Kemmerling, B., Schulze-Lefert, P., Somerville, S. C., and Panstruga, R. 2006. Conserved requirement for a plant host cell protein in powdery mildew pathogenesis. Nat. Genet. 38: 716-720.

Cook, D. E., Mesarich, C. H., and Thomma, B. P. H. J. 2015. Understanding plant immunity as a surveillance system to detect invasion. Annu. Rev. Phytopathol. 53:541-563.

de Torres Zabala, M., Littlejohn, G., Jayaraman, S., Studholme, D., Bailey, T., Lawson, T., Tillich, M., Licht, D., Bölter, B., Delfino, L., Truman, W., Mansfield, J., Smirnoff, N., and Grant, M. 2015. Chloroplasts play a central role in plant defence and are targeted by pathogen effectors. Nat. Plants 1:15074

De Vleesschauwer, D., Xu, J., and Höfte, M. 2014. Making sense of hormone-mediated defense networking: From rice to Arabidopsis. Front. Plant Sci. 5:611.

Dean, J. V., and Delaney, S. P. 2008. Metabolism of salicylic acid in wildtype, ugt74f1 and ugt74f 2 glucosyltransferase mutants of Arabidopsis thaliana. Physiol. Plant. 132:417-425.

Dobin, A., Davis, C. A., Schlesinger, F., Drenkow, J., Zaleski, C., Jha, S., Batut, P., Chaisson, M., and Gingeras, T. R. 2013. STAR: Ultrafast universal RNA-seq aligner. Bioinformatics 29:15-21.
Dussart, F., Douglas, R., Sjökvist, E., Hoebe, P. N., Spoel, S. H., and McGrann, G. R. D. 2018. Genome-based discovery of polyketidederived secondary metabolism pathways in the barley pathogen Ramularia collo-cygni. Mol. Plant-Microbe Interact. 31:962-975.

Eulgem, T. 2006. Dissecting the WRKY web of plant defense regulators. PLoS Pathog. 2:e126.

Ewels, P., Magnusson, M., Lundin, S., and Käller, M. 2016. MultiQC: Summarize analysis results for multiple tools and samples in a single report. Bioinformatics 32:3047-3048.

Fesel, P. H., and Zuccaro, A. 2016. Dissecting endophytic lifestyle along the parasitism/mutualism continuum in Arabidopsis. Curr. Opin. Microbiol. 32:103-112.

Grant, M. R., and Jones, J. D. G. 2009. Hormone (dis)harmony moulds plant health and disease. Science 324:750-752.

Haas, B. J., Papanicolaou, A., Yassour, M., Grabherr, M., Blood, P. D., Bowden, J., Couger, M. B., Eccles, D., Li, B., Lieber, M., MacManes, M. D., Ott, M., Orvis, J., Pochet, N., Strozzi, F., Weeks, N., Westerman, R., William, T., Dewey, C. N., Henschel, R., LeDuc, R. D., Friedman, N., and Regev, A. 2013. De novo transcript sequence reconstruction from RNA-seq using the Trinity platform for reference generation and analysis. Nat. Protoc. 8:1494-1512.

Havis, N., Fountaine, J., Gorniak, K., Paterson, L., and Taylor, J. 2015a. Diagnosis of Ramularia collo-cygni and Rhynchosporium spp. in Barley. Methods Mol. Biol. 1302:29-36.

Havis, N. D., Brown, J. K., Clemente, G., Frei, P., Jedryczka, M., Kaczmarek, J., Kaczmarek, M., Matusinsky, P., McGrann, G. R., Pereyra, S., Piotrowska, M., Sghyer, H., Tellier, A., and Hess, M. 2015b. Ramularia collo-cygni-An emerging pathogen of barley crops. Phytopathology 105:895-904.

Havis, N. D., Oxley, S. P., Piper, S. R., and Langrell, S. R. 2006. Rapid nested PCR-based detection of Ramularia collo-cygni direct from barley. FEMS Microbiol. Lett. 256:217-223.

Heiser, I., Hess, M., Schmidtke, K. U., Vogler, U., Miethbauer, S., and Liebermann, B. 2004. Fatty acid peroxidation by rubellin B, C and D, phytotoxins produced by Ramularia collo-cygni (Sutton et Waller). Physiol. Mol. Plant Pathol. 64:135-143.

Heiser, I., Sachs, E., and Liebermann, B. 2003. Photodynamic oxygen activation by rubellin $\mathrm{D}$, a phytotoxin produced by Ramularia collocygni (Sutton et Waller). Physiol. Mol. Plant Pathol. 62:29-36.

Hjortshøj, R. L., Ravnshøj, A. R., Nyman, M., Orabi, J., Backes, G., Pinnschmidt, H., Havis, N., Stougaard, J., and Stukenbrock, E. H 2013. High levels of genetic and genotypic diversity in field populations of the barley pathogen Ramularia collo-cygni. Eur. J. Plant Pathol. 136: 51-60.

Hoff, K. J., Lange, S., Lomsadze, A., Borodovsky, M., and Stanke, M. 2016. BRAKER1: Unsupervised RNA-seq-based genome annotation with GeneMark-ET and AUGUSTUS. Bioinformatics 32:767-769.

Horton, P., Park, K. J., Obayashi, T., Fujita, N., Harada, H., Adams-Collier, C. J., and Nakai, K. 2007. WoLF PSORT: protein localization predictor. Nucleic Acids Res. 35:W585-W587.

Hurley, B., Subramaniam, R., Guttman, D. S., and Desveaux, D. 2014 Proteomics of effector-triggered immunity (ETI) in plants. Virulence 5: 752-760.

Huss, H. 2004. The biology of Ramularia collo-cygni in meeting the challenges of barley blights. The Second International Workshop on Barley Leaf Blights (ICARDA), April 2002, Aleppo, Syria. http://www. icarda.org/.

Ishiga, Y., Watanabe, M., Ishiga, T., Tohge, T., Matsuura, T., Ikeda, Y., Hoefgen, R., Fernie, A. R., and Mysore, K. S. 2017. The SAL-PAP chloroplast retrograde pathway contributes to plant immunity by regulating glucosinolate pathway and phytohormone signaling. Mol. Plant-Microbe Interact. 30:829-841.

Jansen, C., von Wettstein, D., Schäfer, W., Kogel, K. H., Felk, A., and Maier, F. J. 2005. Infection patterns in barley and wheat spikes inoculated with wild-type and trichodiene synthase gene disrupted Fusarium graminearum. Proc. Natl. Acad. Sci. U.S.A. 102:16892-16897.

Jarosch, B., Kogel, K. H., and Schaffrath, U. 1999. The ambivalence of the barley Mlo locus: Mutations conferring resistance against powdery mildew (Blumeria graminis f. sp. hordei) enhance susceptibility to the rice blast fungus Magnaporthe grisea. Mol. Plant-Microbe Interact. 12: 508-514.

Jones, J. D., and Dangl, J. L. 2006. The plant immune system. Nature 444: 323-329.

Jones, P., Binns, D., Chang, H. Y., Fraser, M., Li, W., McAnulla, C., McWilliam, H., Maslen, J., Mitchell, A., Nuka, G., Pesseat, S., Quinn, A. F., Sangrador-Vegas, A., Scheremetjew, M., Yong, S. Y., Lopez, R. and Hunter, S. 2014. InterProScan 5: Genome-scale protein function classification. Bioinformatics 30:1236-1240. 
Kaczmarek, M., Piotrowska, M. J., Fountaine, J. M., Gorniak, K., McGrann, G. R. D., Armstrong, A., Wright, K. M., Newton, A. C., and Havis, N. D. 2017. Infection strategy of Ramularia collo-cygni and development of ramularia leaf spot on barley and alternative graminaceous hosts. Plant Pathol. 66:45-55.

Kellner, R., Bhattacharyya, A., Poppe, S., Hsu, T. Y., Brem, R. B., and Stukenbrock, E. H. 2014. Expression profiling of the wheat pathogen Zymoseptoria tritici reveals genomic patterns of transcription and hostspecific regulatory programs. Genome Biol. Evol. 6:1353-1365.

Kim, M. C., Panstruga, R., Elliott, C., Müller, J., Devoto, A., Yoon, H. W., Park, H. C., Cho, M. J., and Schulze-Lefert, P. 2002. Calmodulin interacts with MLO protein to regulate defence against mildew in barley. Nature 416:447-451.

Kucharewicz, W., Distelfeld, A., Bilger, W., Müller, M., Munné-Bosch, S., Hensel, G., and Krupinska, K. 2017. Acceleration of leaf senescence is slowed down in transgenic barley plants deficient in the DNA/RNAbinding protein WHIRLY1. J. Exp. Bot. 68:983-996.

Kumar, A. S., Park, E., Nedo, A., Alqarni, A., Ren, L., Hoban, K., Modla, S., McDonald, J. H., Kambhamettu, C., Dinesh-Kumar, S. P., and Caplan, J. L. 2018. Stromule extension along microtubules coordinated with actin-mediated anchoring guides perinuclear chloroplast movement during innate immunity. eLife 7:7.

Kumar, J., Hückelhoven, R., Beckhove, U., Nagarajan, S., and Kogel, K. H. 2001. A compromised Mlo pathway affects the response of barley to the necrotrophic fungus Bipolaris sorokiniana (Teleomorph: Cochliobolus sativus) and its toxins. Phytopathology 91:127-133.

Lee, S. H., Han, Y. K., Yun, S. H., and Lee, Y. W. 2009. Roles of the glyoxylate and methylcitrate cycles in sexual development and virulence in the cereal pathogen Gibberella zeae. Eukaryot. Cell 8:1155-1164.

Leete, E., and Marion, L. 1953. The biogenesis of alkaloids. 7. The FORMATION OF HORDENINE and N-methyltyramine from tyrosine in barley. Can. J. Chem. 31:126-128.

Lewis, J. D., Wan, J., Ford, R., Gong, Y., Fung, P., Nahal, H., Wang, P. W., Desveaux, D., and Guttman, D. S. 2012. Quantitative interactor screening with next-generation sequencing (QIS-Seq) identifies Arabidopsis thaliana MLO2 as a target of the Pseudomonas syringae type III effector HopZ2. BMC Genomics 13:8.

Li, B., and Dewey, C. N. 2011. RSEM: Accurate transcript quantification from RNA-Seq data with or without a reference genome. BMC Bioinformatics 12:323.

Li, B., Meng, X., Shan, L., and He, P. 2016. Transcriptional regulation of pattern-triggered immunity in plants. Cell Host Microbe 19:641-650.

Li, H., Handsaker, B., Wysoker, A., Fennell, T., Ruan, J., Homer, N., Marth, G., Abecasis, G., Durbin, R., and 1000 Genome Project Data Processing Subgroup. 2009. The sequence alignment/map format and SAMtools. Bioinformatics 25:2078-2079.

Linthorst, H. J. M., and Van Loon, L. C. 1991. Pathogenesis-related proteins of plants. Crit. Rev. Plant Sci. 10:123-150.

Liu, J. J., Sturrock, R., and Ekramoddoullah, A. K. 2010. The superfamily of thaumatin-like proteins: Its origin, evolution, and expression towards biological function. Plant Cell Rep. 29:419-436.

Lomsadze, A., Burns, P. D., and Borodovsky, M. 2014. Integration of mapped RNA-Seq reads into automatic training of eukaryotic gene finding algorithm. Nucleic Acids Res. 42:e119.

Lorenz, M. C., and Fink, G. R. 2001. The glyoxylate cycle is required for fungal virulence. Nature 412:83-86.

Lovett, J. V., and Hoult, A. H. C. 1995. Allelopathy and self-defense in barley. Allelopathy 582:170-183.

Lyngkjaer, M. F., Carver, T. L. W., and Zeyen, R. J. 1997. Suppression of resistance to Erysiphe graminis f. sp. hordei conferred by the mlo5 barley powdery mildew resistance gene. Physiol. Mol. Plant Pathol. 50:17-36.

Ma, X., Keller, B., McDonald, B. A., Palma-Guerrero, J., and Wicker, T. 2018. Comparative transcriptomics reveals how wheat responds to infection by Zymoseptoria tritici. Mol. Plant-Microbe Interact. 31: 420-431.

Makepeace, J. C., Oxley, S. J. P., Havis, N. D., Hackett, R., Burke, J. I., and Brown, J. K. M. 2007. Associations between fungal and abiotic leaf spotting and the presence of mlo alleles in barley. Plant Pathol. 56: 934-942.

Mann, J. D., and Mudd, S. H. 1963. Alkaloids and plant metabolism. IV The tyramine methylpherase of barley roots. J. Biol. Chem. 238:381-385.

Mascher, M., Gundlach, H., Himmelbach, A., Beier, S., Twardziok, S. O., Wicker, T., Radchuk, V., Dockter, C., Hedley, P. E., Russell, J., Bayer, M., Ramsay, L., Liu, H., Haberer, G., Zhang, X. Q., Zhang, Q., Barrero, R. A., Li, L., Taudien, S., Groth, M., Felder, M., Hastie, A., Šimková, H., Staňková, H., Vrána, J., Chan, S., Muñoz-Amatriaín, M., Ounit, R., Wanamaker, S., Bolser, D., Colmsee, C., Schmutzer, T., AliyevaSchnorr, L., Grasso, S., Tanskanen, J., Chailyan, A., Sampath, D.,
Heavens, D., Clissold, L., Cao, S., Chapman, B., Dai, F., Han, Y., Li, H., Li, X., Lin, C., McCooke, J. K., Tan, C., Wang, P., Wang, S., Yin, S., Zhou, G., Poland, J. A., Bellgard, M. I., Borisjuk, L., Houben, A., Doležel, J., Ayling, S., Lonardi, S., Kersey, P., Langridge, P., Muehlbauer, G. J., Clark, M. D., Caccamo, M., Schulman, A. H., Mayer, K. F. X., Platzer, M., Close, T. J., Scholz, U., Hansson, M., Zhang, G., Braumann, I., Spannagl, M., Li, C., Waugh, R., and Stein, N. 2017. A chromosome conformation capture ordered sequence of the barley genome. Nature 544:427-433.

McGrann, G. R., Andongabo, A., Sjökvist, E., Trivedi, U., Dussart, F., Kaczmarek, M., Mackenzie, A., Fountaine, J. M., Taylor, J. M., Paterson, L. J., Gorniak, K., Burnett, F., Kanyuka, K., Hammond-Kosack, K. E., Rudd, J. J., Blaxter, M., and Havis, N. D. 2016. The genome of the emerging barley pathogen Ramularia collo-cygni. BMC Genomics 17: 584.

McGrann, G. R., Stavrinides, A., Russell, J., Corbitt, M. M., Booth, A., Chartrain, L., Thomas, W. T., and Brown, J. K. 2014. A trade off between mlo resistance to powdery mildew and increased susceptibility of barley to a newly important disease, Ramularia leaf spot. J. Exp. Bot. 65: 1025-1037.

Miethbauer, S., Heiser, I., and Liebermann, B. 2003. The phytopathogenic fungus Ramularia collo-cygni produces biologically active rubellins on infected barley leaves. J. Phytopathol. 151:665-668.

Mulder, N., and Apweiler, R. 2007. InterPro and InterProScan: Tools for protein sequence classification and comparison. Methods Mol. Biol. 396:59-70.

Muria-Gonzalez, M. J., Chooi, Y. H., Breen, S., and Solomon, P. S. 2015. The past, present and future of secondary metabolite research in the Dothideomycetes. Mol. Plant Pathol. 16:92-107.

Noctor, G., and Foyer, C. H. 1998. Ascorbate and glutathione: Keeping active oxygen under control. Annu. Rev. Plant Physiol. Plant Mol. Biol. 49:249-279.

Palma-Guerrero, J., Torriani, S. F. F., Zala, M., Carter, D., Courbot, M., Rudd, J. J., McDonald, B. A., and Croll, D. 2016. Comparative transcriptomic analyses of Zymoseptoria tritici strains show complex lifestyle transitions and intraspecific variability in transcription profiles. Mol. Plant Pathol. 17:845-859.

Powell, J. J., Carere, J., Sablok, G., Fitzgerald, T. L., Stiller, J., Colgrave, M. L., Gardiner, D. M., Manners, J. M., Vogel, J. P., Henry, R. J., and Kazan, K. 2017. Transcriptome analysis of brachypodium during fungal pathogen infection reveals both shared and distinct defense responses with wheat. Sci. Rep. 7:17212.

Robinson, M. D., McCarthy, D. J., and Smyth, G. K. 2010. edgeR: A Bioconductor package for differential expression analysis of digital gene expression data. Bioinformatics 26:139-140.

Rudd, J. J., Kanyuka, K., Hassani-Pak, K., Derbyshire, M., Andongabo, A., Devonshire, J., Lysenko, A., Saqi, M., Desai, N. M., Powers, S. J., Hooper, J., Ambroso, L., Bharti, A., Farmer, A., Hammond-Kosack, K. E., Dietrich, R. A., and Courbot, M. 2015. Transcriptome and metabolite profiling of the infection cycle of Zymoseptoria tritici on wheat reveals a biphasic interaction with plant immunity involving differential pathogen chromosomal contributions and a variation on the hemibiotrophic lifestyle definition. Plant Physiol. 167:1158-1185.

Salamati, S., and Reitan, L. 2006. Ramularia collo-cygni on spring barley, an overview of its biology and epidemiology. 1st European Ramularia Workshop, Göttingen, Germany.

Sánchez-Vallet, A., Mesters, J. R., and Thomma, B. P. H. J. 2015. The battle for chitin recognition in plant-microbe interactions. FEMS Microbiol. Rev. 39:171-183.

Schulz, B., and Boyle, C. 2005. The endophytic continuum. Mycol. Res. 109:661-686.

Stabentheiner, E., Minihofer, T., and Huss, H. 2009. Infection of barley by Ramularia collo-cygni: Scanning electron microscopic investigations. Mycopathologia 168:135-143.

Su, J., Yang, L., Zhu, Q., Wu, H., He, Y., Liu, Y., Xu, J., Jiang, D., and Zhang, S. 2018. Active photosynthetic inhibition mediated by MPK3/MPK6 is critical to effector-triggered immunity. PLoS Biol. 16:e2004122.

Sutton, B., and Waller, J. 1988. Taxonomy of Ophiocladium hordei causing leaf lesions on Triticale and other Gramineae. Trans. Br. Mycol. Soc. 90:55-61.

Taylor, J. M., Paterson, L. J., and Havis, N. D. 2010. A quantitative realtime PCR assay for the detection of Ramularia collo-cygni from barley (Hordeum vulgare). Lett. Appl. Microbiol. 50:493-499.

Thirugnanasambandam, A., Wright, K. M., Havis, N., Whisson, S. C., and Newton, A. C. 2011. Agrobacterium-mediated transformation of the barley pathogen Ramularia collo-cygni with fluorescent marker tags and live tissue imaging of infection development. Plant Pathol. 60:929-937.

Treutter, D. 2005. Significance of flavonoids in plant resistance and enhancement of their biosynthesis. Plant Biol Stuttg 7:581-591. 
Trezzini, G. F., Horrichs, A., and Somssich, I. E. 1993. Isolation of putative defense-related genes from Arabidopsis thaliana and expression in fungal elicitor-treated cells. Plant Mol. Biol. 21:385-389.

Vanholme, R., Demedts, B., Morreel, K., Ralph, J., and Boerjan, W. 2010. Lignin biosynthesis and structure. Plant Physiol. 153:895-905.

Walters, D. R., Havis, N. D., and Oxley, S. J. 2008. Ramularia collo-cygni: The biology of an emerging pathogen of barley. FEMS Microbiol. Lett. 279:1-7.

Wang, L., Wang, S., and Li, W. 2012. RSeQC: Quality control of RNAseq experiments. Bioinformatics 28:2184-2185.

Yang, G., Zhou, R., Tang, T., and Shi, S. 2008. Simple and efficient isolation of high-quality total RNA from Hibiscus tiliaceus, a mangrove associate and its relatives. Prep. Biochem. Biotechnol. 38:257-264.

Zeilinger, S., Gupta, V. K., Dahms, T. E., Silva, R. N., Singh, H. B., Upadhyay, R. S., Gomes, E. V., Tsui, C. K., and Nayak S, C. 2016. Friends or foes? Emerging insights from fungal interactions with plants. FEMS Microbiol. Rev. 40:182-207.
Zhang, L., Du, L., and Poovaiah, B. W. 2014. Calcium signaling and biotic defense responses in plants. Plant Signal. Behav. 9:e973818.

Zhou, Z., Takaya, N., Nakamura, A., Yamaguchi, M., Takeo, K., and Shoun, H. 2002. Ammonia fermentation, a novel anoxic metabolism of nitrate by fungi. J. Biol. Chem. 277:1892-1896.

Zipfel, C., and Oldroyd, G. E. 2017. Plant signalling in symbiosis and immunity. Nature 543:328-336.

\section{AUTHOR-RECOMMENDED INTERNET RESOURCES}

Gene Ontology Consortium website: geneontology.org GPI-anchor Predictor datasets: http://gpcr2.biocomp.unibo.it/predgpi/dataset.htm PHI-base: http://www.phi-base.org 